\title{
Do alerting signals increase the size of the attentional focus?
}

\author{
Verena C. Seibold ${ }^{1}$
}

Published online: 22 November 2017

(C) The Psychonomic Society, Inc. 2017

\begin{abstract}
Previous research has shown that the presentation of an auditory alerting signal before a visual target increases the interference from flanking distractors. Recently, it has been suggested that this increase in interference may be due to an expansion of the spatial focus of attention. In five experiments, this hypothesis was tested by using a probe technique dedicated to measuring variations in the size of the attentional focus: In the majority of trials, participants performed a letter discrimination task in which their attention was focused on a central target letter. In a randomly intermixed probe task, the size of the attentional focus was measured by letting participants respond to a probe occurring at varying positions. In all experiments, reaction time (RT) to the probe increased from the most central to more lateral probe positions. This V-shaped probe-RT function, however, was not flattened by the presentation of an alerting signal. Overall, this pattern of results is inconsistent with the hypothesis that alerting signals increase the attentional focus. Instead, it is consistent with nonspatial accounts that attribute the increase in interference to an alerting effect on perceptual processing, which then leads to a detrimental effect at the level of response selection.
\end{abstract}

Keywords Attention $\cdot$ Executive control $\cdot$ Perception and action

Among the basic cognitive mechanisms that enable humans to understand the world and act in a flexible and goal-directed manner, attention is presumably the most prominent one.

Verena C. Seibold

verena.seibold@uni-tuebingen.de

1 Evolutionary Cognition, Department of Psychology, University of Tübingen, Schleichstraße 4, D-72076 Tübingen, Germany
Already in 1890, William James stated that attention "is taking possession of the mind . . . of one out of what seem several simultaneously possible objects or trains of thought" (James, 1890, p. 403). Since then, cognitive psychologists have studied extensively the way in which attention privileges some over other information (for reviews, see, e.g., Carrasco, 2011; Driver, 2001; Raz \& Buhle, 2006).

As one of the key insights, it has been recognized that attention operates on information processing via several distinct mechanisms that may serve different functions. For instance, according to one influential theory (Petersen \& Posner, 2012; Posner \& Boies, 1971; Posner \& Petersen, 1990; see also Raz \& Buhle, 2006), attention entails three major functions that are organized in different neuronal networks (for an overview of these networks, see, e.g., Petersen \& Posner, 2012; Raz \& Buhle, 2006): First of all, attention serves the maintenance of an alert state (alerting function) by activating processing resources that can then be flexibly allocated to different processing requirements. Evidence for this function comes from various studies showing that the presentation of a noninformative auditory alerting signal (AS) shortly before a response-relevant stimulus speeds up reaction time (RT) to that stimulus (e.g., Bernstein, Clark, \& Edelstein, 1969; Hackley \& Valle-Inclán, 1998; Keuss, 1972; Ulrich \& Mattes, 1996). Second, attention also serves the direction of processing resources toward a specific stimulus, location or task (orienting function). This orienting function is most apparent in the spatial cueing paradigm, in which a cue either correctly (validly) or incorrectly (invalidly) announces the location of a subsequent target. It is generally assumed that attention is directed toward the cued location, and this leads to a processing advantage if the target is presented at that location. In line with this assumption, performance is better for validly cued targets than for invalidly cued targets (e.g., Posner, 1980; Posner, Snyder, \& Davidson, 1980). Finally, 
attention also serves the resolution of conflict or interference that arises when relevant and irrelevant (or even distracting) pieces of information compete for processing resources (focal attention or executive control function). This control function of attention is best illustrated in congruency effects that are typically observed in conflict paradigms such as the Eriksen flanker task (Eriksen \& Eriksen, 1974), the Stroop task (Stroop, 1935), and the Simon task (Simon \& Rudell, 1967). In these tasks, a task-irrelevant stimulus or stimulus feature overlaps with a task-relevant stimulus or the response to the task-relevant stimulus. For instance, in the flanker task, a target is surrounded by task-irrelevant distractor letters (flankers), which map either to the same response as the target (congruent) or to the opposite response (incongruent). Similarly, in the Stroop task, the task-irrelevant semantic content of a color word is either the same as the word's color (e.g., "red" written in red) or different from it (e.g., "green" written in red). Finally, in the Simon task, the task-irrelevant location of a laterally presented target (e.g., right) either does or does not correspond to the location of the response effector (e.g., right hand). In all cases, the activation of the irrelevant dimension (i.e., the response indicated by the flankers in the flanker task, the semantic content in the Stroop task, or the target location in the Simon task) leads to a processing conflict in the incongruent condition, as reflected in longer RTs and a higher error rate. The ability of the cognitive system to reduce or even prevent this processing conflict is typically regarded as an index of the efficiency of executive control.

Despite the theoretical and neurophysiological distinction between the alerting, orienting, and executive control systems, experimental work has provided compelling evidence that these subsystems do not operate independently on information processing, but rather interact substantially in their effects (see, e.g., Callejas, Lupiáñez, Funes, \& Tudela, 2005; Callejas, Lupiáñez, \& Tudela, 2004; Fan, McCandliss, Sommer, Raz, \& Posner, 2002; Weinbach \& Henik, 2012; but see Fernandez-Duque \& Posner, 1997). Of these interactions, the most striking one has been reported between the alerting system and executive control: Specifically, it has been shown that alerting signals - despite their beneficial effect on RT - increase congruency effects. This congruency-byalerting interaction has been demonstrated predominantly in different variants of the flanker task (Callejas et al., 2004; Fischer, Plessow, \& Kiesel, 2012; Weinbach \& Henik, 2012) and the Simon task (Böckler, Alpay, \& Stürmer, 2011; Fischer, Plessow, \& Kiesel, 2010). Furthermore, studies including a neutral comparison condition suggest that this interaction, at least in the flanker task, is driven mainly by an alerting effect on the incongruent rather than the congruent condition (e.g., Fan et al., 2002; Habekost, Petersen, \& Vangkilde, 2014; Weinbach \& Henik, 2012). This pattern of results indicates that alerting signals exert a detrimental effect on stimulus processing in a situation of conflict.
Not surprisingly, various accounts have been proposed in order to explain this detrimental effect. Originally, it was assumed that the alerting effect directly reflects an inhibitory effect on executive control (Callejas et al., 2005; Callejas et al., 2004). This account is based on the idea that the main function of the alerting system is to enhance the speed of responding toward a stimulus (e.g., Posner, 1994). To guarantee this state of high responsiveness, the alerting system inhibits activation of the executive control system and thereby prevents the time-consuming in-depth analysis of a stimulus. This idea fits nicely with the finding that the congruency-byalerting interaction in the flanker task is driven mainly by the incongruent condition, because this condition requires deeper, controlled stimulus processing in order to overcome the conflict between the relevant and irrelevant stimulus dimensions.

The inhibition account, however, has been questioned by authors who argue that alerting signals do not affect executive control in a direct, but rather in an indirect, manner, by facilitating automatic response activation (Böckler et al., 2011; Fischer et al., 2010, 2012; Fischer, Schubert, \& Liepelt, 2007). This account is rooted in the assumption that response selection is driven by information accumulation along two routes (see, e.g., De Jong, Liang, \& Lauber, 1994): a controlled route, which accumulates information about the response corresponding to the task instruction, and an automatic route, which accumulates information on the basis of direct stimulus-response bindings. These stimulus-response bindings are established, for instance, through repeated pairing of a stimulus with a response. Importantly, such bindings are assumed to be activated automatically, even when the stimulus is task-irrelevant. On the basis of this dual-route architecture, it has been proposed that alerting signals increase the rate of information accumulation in the automatic route (e.g., Fischer et al., 2010), and thereby facilitate the activation of the corresponding response. Consequently, the response activated in the automatic route will have a stronger impact on the response decision and, in the case of incongruent information, lead to a response conflict. In support of this automatic response activation account, Böckler et al. (2011) showed that alerting signals not only increase congruency effects in the Simon task, but also increase the amplitude of the positive lateralized readiness potential (LRP), an event-related potential marker of automatic response activation (e.g., Eimer, 1995). Furthermore, evidence supporting the response activation account has been reported by Fischer et al. (2012). These authors used a modified version of the flanker task in which a target (a pleasant or unpleasant word) was paired with either identical flankers - that is, words that were also presented as targets, and therefore were associated with a response- or a so-called category flankers - that is, words that were never presented as targets, but belonged to the same semantic category. Although Fischer et al. (2012) observed congruency effects in both flanker conditions, an interaction with alerting 
signals emerged only in the identical flanker condition, not in the category flanker condition. This pattern of results is compatible with the response activation account, because this account specifically predicts a congruency-by-alerting interaction only for flankers that are associated with a response. It is incompatible, however, with the inhibition account, because this account predicts a congruency-by-alerting interaction irrespective of whether or not the flankers are associated with a response. To summarize, the results of Böckler et al. (2011) and Fischer et al. (2012) clearly suggest an involvement of automatic response activation in the alerting effect.

A third major account was proposed more recently by Weinbach and Henik (2012). These authors conducted an elegant series of experiments in which they tested both the inhibition and the response activation account. Specifically, in one experiment (Exp. 2 of Weinbach \& Henik, 2012), they examined the inhibition account by measuring the effect of alerting signals on conflict in the classical Stroop task. The conflict between the color and the semantics of a word, as measured in the Stroop task, is typically regarded as a direct index of the ability to inhibit a task-irrelevant feature (e.g., Diamond, 2013). Correspondingly, Weinbach and Henik (2012) reasoned that an inhibitory effect of alerting on executive control should show up in an increased Stroop effect. In contrast to this prediction, however, the authors did not observe any modulation of the Stroop effect by alerting signals. In two subsequent experiments (Exps. 3 and 4), Weinbach and Henik (2012) then tested the response activation account using two variants of the classical flanker task. Specifically, in both experiments participants performed either a left- or a right-hand response, depending on the color of a centrally presented target. In one experiment (Exp. 3), the target itself was an arrow pointing to the left or right, whereas in the other experiment (Exp. 4), the target was simply a patch that was surrounded by arrow flankers pointing to the left or right. Therefore, the task-irrelevant dimension (the orientation of the arrow) was either part of the target itself or spatially separated from it. Importantly, Weinbach and Henik (2012) showed that the congruency-by-alerting interaction only emerged when the irrelevant dimension was spatially separated from the target, not when it was spatially integrated into the target. According to the authors, this result can hardly be reconciled with a pure response activation account, because such an account would predict larger congruency effects irrespective of whether the relevant and irrelevant dimensions were presented at the same or at different spatial locations. Weinbach and Henik (2012) therefore concluded that the involvement of spatial attention may be an important factor in observing the congruency-by-alerting interaction.

On the basis of these considerations, Weinbach and Henik (2012) proposed a spatial hypothesis, which attributes the congruency-by-alerting interaction to an influence on spatial attention. More specifically, they hypothesized that alerting signals expand the focus of visuospatial attention and thereby increase the accessibility of events in the spatial surrounding of the target. In contrast to previous accounts, this spatial hypothesis provides a straightforward explanation for the observation that alerting signals increase congruency effects in the flanker task only when the relevant and irrelevant dimensions are spatially separated (Exp. 4 of Weinbach \& Henik, 2012). That is, an expansion of the spatial focus should specifically facilitate the processing of stimuli in the vicinity of the target, whereas it should not affect processing of the target itself. Furthermore, this hypothesis can also explain the observation of a larger congruency effect in the Simon task (Böckler et al., 2011; Fischer et al., 2010), since a broader attentional beam would also enhance the likelihood that the (irrelevant) location of the target would be processed and thereby affect responding to the relevant feature. Finally, this account is also supported by some empirical evidence. Specifically, the account can also explain the results of a study by Weinbach and Henik (2011), who investigated the effect of alerting signals on conflict in a global-local task. In this kind of task, participants have to respond to either the global or the local feature of a hierarchical stimulus, which either conveys identical (congruent) or conflicting (incongruent) feature information. For instance, Weinbach and Henik (2011) presented large arrowheads pointing to the left or right, which were composed of small arrowheads also pointing to the left or right. Importantly, they showed that alerting signals specifically increased interference arising from the global feature, whereas the interference arising from the local feature remained unaffected (but see Weinbach \& Henik, 2014). Again, this result is consistent with an interpretation in terms of a larger focus of spatial attention, since a larger focus should increase the likelihood of a global stimulus interpretation.

Like the inhibition account and the response selection account, the spatial account also fails to explain some of the abovementioned empirical findings. Most notably, this account cannot explain in a straightforward manner why alerting signals increase congruency effects in a flanker task when using identical stimuli for the flankers and targets, but not when using flankers that are only from the same semantic category as the target (Fischer et al., 2012). Specifically, on the basis of the notion that spatial attention operates at an early level of stimulus processing - that is, before stimulus identification - it should not matter whether the flankers are identical to potential targets or whether they are only categorically related to the target. Instead, an expansion of the attentional focus should affect all stimuli near the target, irrespective of their semantic identity.

To summarize, there is clear evidence that alerting signals increase congruency effects in conflict tasks (Böckler et al., 2011; Callejas et al., 2005; Callejas et al., 2004; Fischer et al., 2010, 2012; Weinbach \& Henik, 2012). The mechanisms that 
contribute to this effect, however, are still debated. Although several major accounts have been proposed to explain the effect, the existing empirical evidence regarding these accounts is quite mixed. First of all, although the inhibition account can basically explain why the alerting effect mainly results from the incongruent condition in the flanker task, it cannot explain why alerting signals modulate the congruency effect only if the flankers are directly associated with a response (Fischer et al., 2012). Second, although the response activation account receives direct support from an eventrelated potential study (Böckler et al., 2011), it cannot explain in a straightforward manner why alerting signals do not increase congruency effects when the irrelevant dimension is spatially integrated into the target. Third, the spatial account (Weinbach \& Henik, 2012), according to which alerting signals increase the size of the attentional focus, cannot explain why alerting signals do not modulate congruency effects for categorical flankers (Fischer et al., 2012). Furthermore, and in contrast to the inhibition and response activation accounts, the spatial account has not yet undergone a direct empirical test.

The aim of the present study is to fill in this gap and to investigate whether an alerting signal increases the size of the attentional focus, as is suggested in the spatial account (Weinbach \& Henik, 2012). To measure variations in the size of the attentional focus, a paradigm proposed by LaBerge (1983) was used. In this paradigm, participants perform two kinds of tasks: a main task, in which attention is focused on a centrally presented target, and a probe task, in which the size of the attentional focus is then measured by probing different locations around the target. For instance, in Experiment 1 of LaBerge's study, the main task required participants to focus on a central target letter in a five-letter display and to either respond or not respond, depending on whether the target belonged to a go set or a no-go set of letters. In a subset of trials, a probe was presented instead of the main task display, and participants again had to respond or not respond, depending on the probe's identity. Crucially, in contrast to the target letter in the main task, the probe occurred randomly at all five array positions; thus, the speed of responding to the probe provides an index of how much attention was deployed to the probe's position. In line with this logic, LaBerge observed that RT to the probe as a function of its position exhibits a Vshaped trend: RT is fastest at the central position (i.e., the position where the target is presented in the main task) and increases toward more lateral probe positions. LaBerge could show that the V shape of the probe-RT function can be mathematically described in terms of a quadratic trend. Furthermore, he could show that this quadratic trend is observed only when participants' attention in the main task is focused on the central display position; in contrast, the trend does not emerge when participants' attention is distributed across the whole letter array. For instance, when participants were instructed to respond to the identity of the whole display in the main task, the probe-RT function did not show the quadratic trend anymore, but instead was completely flattened (LaBerge, 1983). This pattern of results, which has been successfully replicated (LaBerge, 1983, Exp. 2; LaBerge \& Brown, 1986), shows that participants can flexibly adjust the size of the attentional focus (according to the position of the task-relevant information), and that this adjustment is reflected in the shape of the probe-RT function. Hence, LaBerge's paradigm provides an effective means to measure variations in the size of the attentional focus.

In the present study, five experiments were conducted based on the LaBerge (1983) paradigm. In Experiment 1, LaBerge's paradigm was combined with an alerting signal, which was presented in half of the trials in the main and the probe task. Experiment 2 was identical to Experiment 1, except that the probe task was presented directly after the main task in order to capture the direct effect of the alerting signal on the main task. In both experiments, neutral letters were used as flankers in the main task, thereby preventing the emergence of any stimulus-response binding between the flanker and the target response. Accordingly, any effect of the alerting signal in these experiments cannot be attributed to automatic response activation, but instead reflects a pure effect on the attentional focus. In Experiments 3 and 4, arrows were used as flankers (analogous to Weinbach \& Henik, 2012, Exp. 4), so that the flankers now contained an irrelevant spatial dimension. Thereby, the experiments investigated whether alerting signals may increase the attentional focus only in the presence of irrelevant spatial information. In the final Experiment 5, letters from the same stimulus set were used as the target and flankers in the main task, so that the flankers were directly associated with the target response. Furthermore, analogous to Experiment 1, the main and the probe task were presented in separate trials, and an alerting signal was presented in half of the trials in both tasks. Thereby, this experiment investigated whether an influence of alerting signals on the attentional focus could be observed in the presence of a strong congruency-by-alerting interaction and a direct alerting effect on processing of the probe.

\section{Experiment 1}

Experiment 1 served as a first test for the hypothesis of an increased focus of spatial attention in alerted conditions. As in the paradigm of LaBerge (1983, Exp. 1), participants performed two tasks: On the majority of trials, they performed a speeded letter discrimination task, in which they had to respond to a central target letter. In a procedure similar to the classical flanker task, the target letter was surrounded by four flanker letters. In contrast to the classical flanker task, however, the flanker letters were never presented as targets. As such, they would not lead to any conflict with the target response, 
but nevertheless would require participants to perform a filtering operation in order to discriminate the target from them. The difficulty of discrimination was further stressed by letting participants perform a go-no-go response, depending on the identity of the target. Overall, this task setup should induce a narrow focus of attention in the main task centered at the target position (see LaBerge, 1983). The size of the attentional focus was then measured in the probe task. Specifically, in some trials a probe was presented instead of the main task display. As in the main task, participants performed a speeded go-nogo response, depending on the identity of the probe. Importantly, the probe occurred randomly at one of the five positions used in the main task, and thus at both the target and flanker positions. The speed of responding to the probe, as indexed by RT, served as a measure of the size of the attentional focus.

To measure the alerting effect on the size of the attentional focus, an alerting signal was presented in half of the trials in both the main and probe tasks. In line with previous studies (e.g., Callejas et al., 2004; Fischer et al., 2012; Weinbach \& Henik, 2012), I expected that the presentation of an alerting signal would lead to an overall reduction of RT in the main and probe tasks. Furthermore, the probe-RT should vary as a function of the probe's position, as indexed by a V-shaped RT function (LaBerge, 1983; LaBerge \& Brown, 1986). Most importantly, this probe-RT function should be affected by the presence of an alerting signal: Specifically, if alerting increases the size of the attentional focus, the processing of more lateral stimuli should specifically benefit from it. Therefore, the $\mathrm{V}$ shape of the probe-RT function (as reflected in a quadratic trend) should be reduced (or even eliminated) in the presence of an alerting signal.

\section{Method}

Participants Thirty-six participants, all students from the local university (23 women, $13 \mathrm{men} ; M_{\text {age }}=21.03$ years, age range: 19-27 years) took part in Experiment 1 . They had normal or corrected-to-normal visual acuity and received either course credit or payment $(€ 10.50)$. All participants gave written informed consent prior to their participation.

Apparatus and stimuli The stimuli were presented via Experimental Runtime System (Berisoft, Frankfurt am Main, Germany) on a standard 22-in. CRT monitor. The viewing distance was approximately $55 \mathrm{~cm}$. Responses were collected via custom-made response keys allowing for precise RT measurement. An auditory sine tone $(1000 \mathrm{~Hz}, 70 \mathrm{~dB})$, presented via headphones, served as alerting signal. All visual stimuli were presented in white (luminance: $70 \mathrm{~cd} / \mathrm{m}^{2}$ ) on a dark background (luminance: $0.01 \mathrm{~cd} / \mathrm{m}^{2}$ ).

A small horizontal line (width: $0.5^{\circ}$ of visual angle, edge thickness: $0.1^{\circ}$ of visual angle) served as fixation line. The main task display consisted of five uppercase letters. The central letter (the target) was presented at the center of the screen (i.e., middle display position). It was randomly drawn from two sets of letters, which were defined as go letters (e.g., the letters A, B, C, D, and E) and no-go letters (e.g., the letters N, $\mathrm{O}, \mathrm{P}, \mathrm{R}$, and $\mathrm{S}$ ). The target was flanked by four letters, which were presented at positions to the left and right (i.e., the positions left, middle-left, middle-right, and right). The four flanker letters were randomly drawn from a letter set containing ten neutral letters (i.e., H, J, K, L, M, T, U, V, X, and Y), which were never presented as target. The probe task display consisted of either the letter " $Z$ " or the digit "7" and four "+" signs as flanking stimuli. The probe stimulus (i.e., $\mathrm{Z}$ vs. 7) was presented randomly at one of the five display locations. All stimuli in the main and probe task were of equal size (height $\times$ width: $0.5^{\circ} \times 0.5^{\circ}$ of visual angle), with an edge thickness of $0.1^{\circ}$ of visual angle. Furthermore, the overall display size in main task and probe task was equal (height $\times$ width: $0.5^{\circ} \times 2.8^{\circ}$ of visual angle).

Procedure The procedure of an experimental trial is illustrated in Fig. 1A. Throughout each trial, the fixation line stayed on screen. A trial started with a random interval of $500+X \mathrm{~ms}$, where $X$ was drawn from an exponential distribution with a mean of $1,000 \mathrm{~ms}$. In trials with an alerting signal, the random interval was followed by the presentation of the alerting signal for $100 \mathrm{~ms}$ and a subsequent interstimulus interval (ISI) of 50 ms. This rather short ISI was chosen to ensure that the alerting effect would mainly be driven by an increase in phasic arousal (see also Weinbach \& Henik, 2013). In trials without an alerting signal, the random interval was followed by an additional interval lasting $150 \mathrm{~ms}$. Then, either the main-task or the probe-task display appeared on screen, either until a participant responded or maximally for $1,500 \mathrm{~ms}$. For the main task, participants were instructed to respond as fast as possible to the central letter if it was part of the go set (e.g., one of the letters A, B, C, D, or E), but not to respond if it was part of the no-go set (e.g., one of the letters N, O, P, R, or S). Similarly, for the probe task, participants were instructed to respond as fast as possible to the go stimulus (e.g., the letter $Z$ ), but not to the no-go stimulus (e.g., the digit 7), irrespective of its position. Participants responded with their right index finger in both the main and the probe task. In the case of an erroneous response, error feedback ("Fehler") was presented for $300 \mathrm{~ms}$ below fixation. The end of each trial was followed by an intertrial interval of 2,000 ms.

Each participant took part in one experimental session, which lasted about $50 \mathrm{~min}$. Participants first completed three practice blocks with 15 trials each, in which they practiced the main task (Block 1), the probe task (Block 2), and both tasks in combination with the alerting signal (Block 3 ). Within practice blocks, participants were given feedback for both correct responses ("Richtig") and erroneous responses ("Fehler"). 


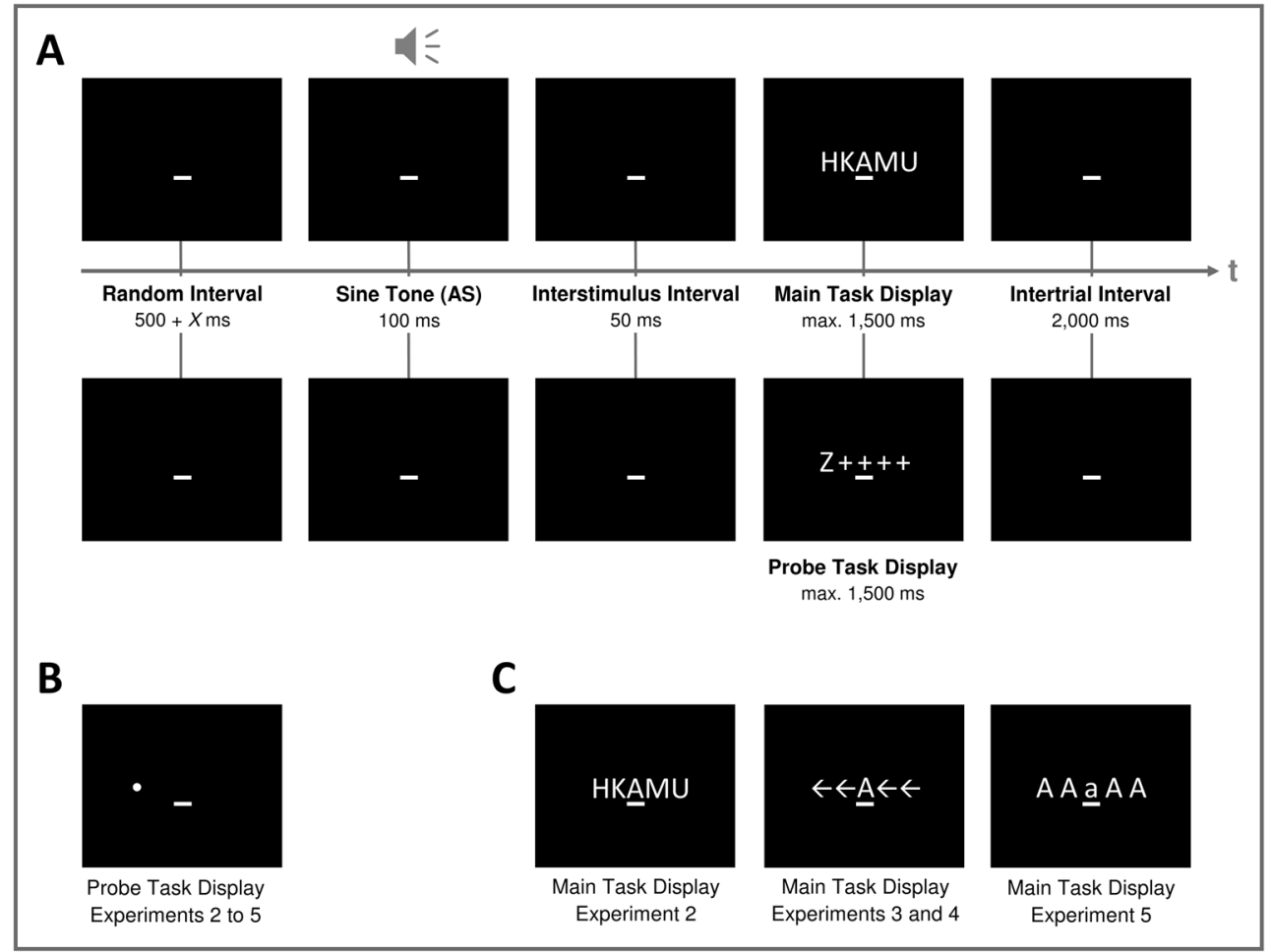

Fig. 1 (A) Major trial events in Experiment 1, illustrated separately for the main task (upper row) and the probe task (lower row). Only trials with an alerting signal (AS) are shown. In Experiment 1, the main task and probe task were presented in separate trials (see LaBerge, 1983). In both tasks, participants performed a speeded go-no-go response that depended on the identity of the central letter or the probe, respectively. (B) Probetask display in Experiments 2-5. In contrast to Experiment 1, the probe was a small white dot, and participants were instructed to respond whenever they detected the probe. (C) Main-task displays in Experiments 2-5.

Afterward, participants completed 700 experimental trials in total, which were subdivided into two blocks of 350 trials. A short break was inserted after each 35 trials. To ensure that participants would primarily prepare for the main task, they were informed that the main task would be presented in the majority of trials. Furthermore, they were given feedback regarding their mean RT and accuracy in the main task within each break. The ratio of main task to the probe task was 5:2, leading to 500 trials for the main task and 200 trials for the probe task. Within each task, the ratio of go to no-go trials was 4:1. Correspondingly, participants completed 400 go trials and 100 no-go trials in the main task, and 160 go trials and 40 nogo trials in the probe task. Half of the trials within each task and block included an alerting signal. The main and probe tasks, as well as trials with and without alerting signals, were randomly intermixed within each experimental block. Furthermore, the designations of the target letters (i.e., A, B, C, D, E vs. N, O, P, R, S) as go versus no-go stimuli in the main task and of the probe stimuli (i.e., $Z$ vs. 7) as go versus no-go stimuli in the probe task were counterbalanced across participants.
In Experiment 2, the main task was the same as in Experiment 1. In Experiments 3-5, a flanker task served as the main task, and participants made a spatial response (i.e., left or right) depending on the identity of a central target letter. In Experiments 3 and 4, the central target letter was an uppercase letter, and the flankers were arrows pointing to the left or right. In Experiment 5, the central target letter was a lowercase letter, and the flankers were uppercase letters from the same set as the target letter.

\section{Results}

The statistical analyses for this and all following experiments were conducted in R (R Development Core Team, 2016) and SPSS (IBM, Ehningen, Germany). Practice trials were discarded from the data analysis. Furthermore, trials with RTs below $150 \mathrm{~ms}$ or above 1,400 ms were considered outliers and excluded from the RT analysis $(0.17 \%$ in the main task and $0.14 \%$ in the probe task). Mean accuracy in go trials was close to ceiling in both tasks $(M=99.8 \%$ in the main task and $M=99.9 \%$ in the probe task) and therefore was not analyzed. Mean accuracy in no-go trials was $M=73.1 \%$ in the main task and $M=73.7 \%$ in the probe task, and was therefore analyzed.

The mean RT for correct responses in go trials and mean accuracy in no-go trials were analyzed separately for the main and probe tasks. For the main task, a repeated measures analysis of variance (rmANOVA) with the factor alerting signal (with alerting signal, without alerting signal) was conducted on mean RT in go trials and mean accuracy in no-go trials. For the probe task, two different analysis approaches were chosen: First, mean RT for correct responses in go trials and mean accuracy in no-go 
trials were analyzed via separate two-way rmANOVA with factors alerting signal (with alerting signal, without alerting signal) and probe position (left, middle-left, middle, middle-right, right). Second, a trend analysis was conducted on probe RT in order to model the $\mathrm{V}$ shape of the probe-RT function. As was mentioned before, this $\mathrm{V}$ shape should be reflected in a quadratic trend in the trend analysis. To account for potential differences between the conditions in overall RT, the probe RT was first standardized according to a method proposed by LaBerge (1983): Specifically, for each experimental block and each alerting signal condition, each participant's mean RT for each probe position was subtracted from the participant's mean RT averaged across probe positions. The resulting difference scores were averaged across blocks and then subjected to a trend analysis (using orthogonal polynomial contrasts), with the factors alerting signal (with, without) and probe position (left, middle-left, middle, middle-right, right).

For this and all following experiments, violations of the sphericity assumption in rmANOVAs are compensated for by reporting Greenhouse-Geisser-corrected $p$-values. Furthermore, $\eta_{\mathrm{p}}{ }^{2}$ is reported for all significant main effects and interactions as a measure of effect size. This measure provides an index for the variance explained by a factor in an rmANOVA when controlling for all other factors. Furthermore, all post-hoc analyses of potential main effects or interactions were performed by means of paired $t$ tests. To compensate for an increase in the Type- I error rate, the corresponding $p$-values were corrected according to the Bonferroni-Holm method (Holm, 1979), and these corrected $p$-values will be denoted as $p_{\text {Holm }}$.

Mean RT in the main and the probe task is illustrated in Fig. 2. The analysis of mean RT in the main task revealed a main effect of alerting signal, $F(1,35)=142.82, p<.001, \eta_{\mathrm{p}}{ }^{2}=.80$, since mean RT in trials with an alerting signal was shorter than that in trials without an alerting signal (see Fig. 2, upper panel). The analysis of mean accuracy in no-go trials did not reveal any difference between main task trials with an alerting signal $(M=71.6 \%)$ and main task trials without an alerting signal $(M=74.6 \%), F(1,35)=2.87, p=.10$. The analysis of mean RTs in the probe task revealed a main effect of alerting signal, $F(1,35)=23.32, p<.001, \eta_{\mathrm{p}}{ }^{2}=.40$. Participants responded faster when an alerting signal preceded the probe display (see Fig. 2, lower panel). Furthermore, a main effect of probe position emerged, $F(1,35)=16.34, p<.001, \eta_{\mathrm{p}}{ }^{2}=.32$, since RT clearly varied as a function of the probe's position. Finally, there was also an interaction of probe position with an alerting signal, $F(4,140)=2.93, p=.02, \eta_{\mathrm{p}}{ }^{2}=.08$. The analysis of mean accuracy in no-go probe trials revealed a main effect of alerting signal, $F(1,35)=5.42, p=.03, \eta_{\mathrm{p}}{ }^{2}=$ .13 , in which participants were less accurate in trials with $(M=$ $71.0 \%)$ than in trials without $(M=76.4 \%)$ an alerting signal. Unexpectedly, a main effect of probe position was also observed, $F(4,140)=3.18, p=.02, \eta_{\mathrm{p}}{ }^{2}=.08$, suggesting that

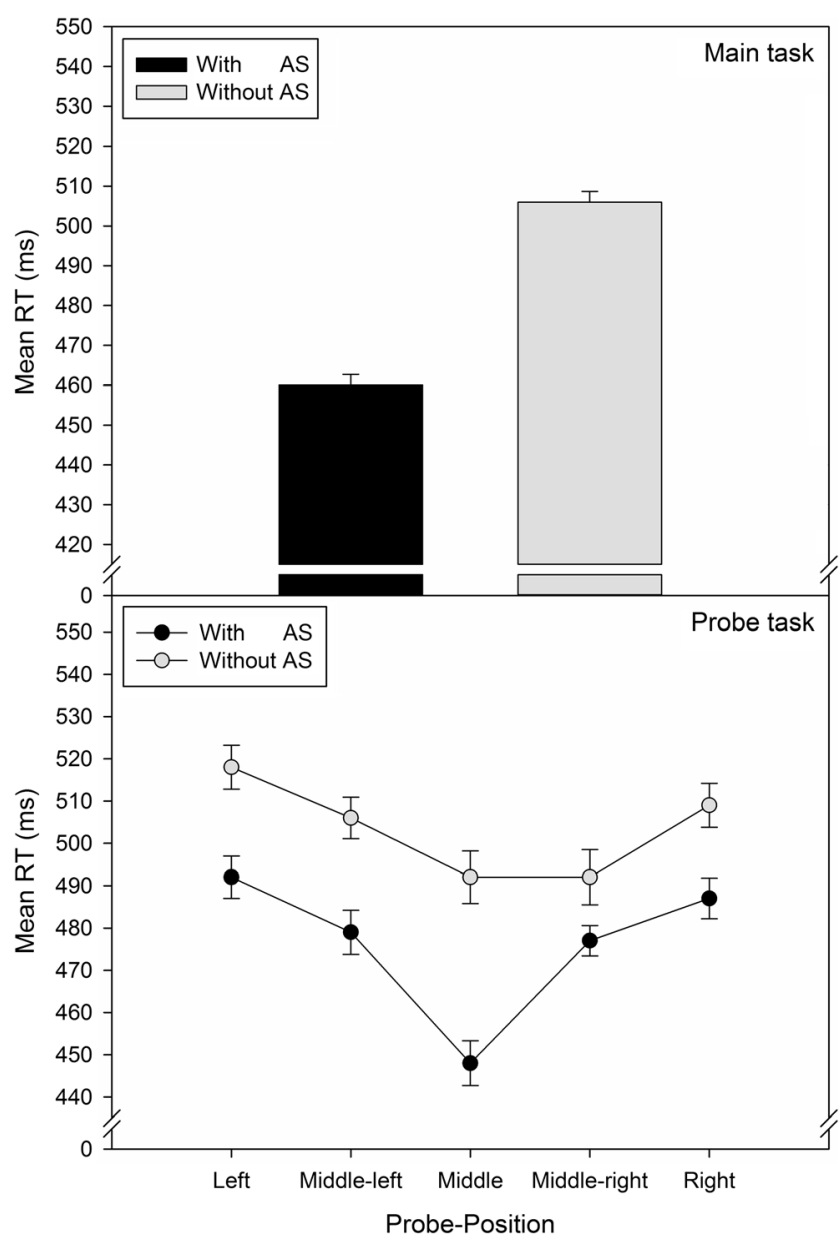

Fig. 2 Results of Experiment 1. (Upper panel) Mean reaction time (RT) in the main task as a function of alerting signal (AS). (Lower panel) Mean $\mathrm{RT}$ in the probe task as a function of AS and probe position. In this and all following figures, error bars represent standard errors for within-subjects designs, as computed according to Morey (2008).

accuracy in no-go trials also varied as a function of the probe's position. There was no interaction of the two factors, $F(4,140)$ $=0.75, p=.56$.

To investigate whether the unexpected variation of accuracy in no-go trials accounted for the shape of the probe-RT function in go trials, additional post-hoc tests were conducted: Specifically, for both dependent measures, the main effect of probe position was analyzed by comparing each two neighboring probe positions. For mean RT in go trials, this posthoc comparison showed that RT increased from the middle toward the middle-left $\left(p_{\text {Holm }}=.001\right)$ and the middle-right position $\left(p_{\text {Holm }}=.01\right)$, and then further increased toward the outward positions (both $p_{\mathrm{Holm}} \mathrm{S}<.005$; see Fig. 2, lower panel). The corresponding analysis on accuracy in no-go trials revealed that the main effect of probe position was due to a drop in accuracy at the middle probe location $(M=67.0 \%)$ when compared to the middle-left $(M=77.1 \%)$ and middleright $\left(\mathrm{M}=78.8 \%\right.$; both $\left.p_{\text {Bolm }} \mathrm{s}<.007\right)$ position. In contrast, there was no accuracy difference between the middle-left and 
left position $(M=72.6 \%)$, nor between the middle-right and right position $\left(M=72.9 \%\right.$; both $\left.p_{\mathrm{Holm}} \mathrm{s}>.29\right)$. Hence, although accuracy in no-go probe trials varied as a function of the probe's position, this effect was specific to the middle probe position.

The trend analysis on standardized probe RT showed several trends within the probe-RT function: First and most importantly, probe RT exhibited a pronounced quadratic trend as a function of probe position, $F(1,35)=47.39, p<.001, \eta_{\mathrm{p}}{ }^{2}=$ .58. As is illustrated in Fig. 2 (lower panel), the probe-RT function had a clear V shape, with the fastest RT for the middle position and increasing RTs toward more lateral positions. Importantly, this quadratic trend was not modulated by alerting signal, $F(1,35)=1.28, p=.27$. Furthermore, there was also a quartic effect of probe position, $F(1,35)=7.19, p=$ $.01, \eta_{\mathrm{p}}{ }^{2}=.17$, and this trend was modulated by alerting signal, $F(1,35)=10.18, p=.003, \eta_{\mathrm{p}}{ }^{2}=.23$. Figure 2 (lower panel) again shows that this effect reflects a specific RT benefit for the middle position in trials with an alerting signal. Finally, the effect of probe position also showed up in a linear trend, $F(1$, $35)=5.71 . p=.02, \eta_{\mathrm{p}}{ }^{2}=.14$, with RT to more rightward probe positions being faster than RT to more leftward positions. No further effects were observed in the trend analysis (all $F \mathrm{~s}<1.5$ ).

To further investigate the nature of the interaction of the alerting signal with the quartic trend in the probe-RT function, I conducted an additional analysis. Specifically, I first calculated the RT difference between each two neighboring probe positions (e.g., left vs. middle-left, middle-left vs. middle, middle vs. middle-right, and middle-right vs. right) and then compared these difference values between trials with and without an alerting signal. This analysis revealed that the RT differences between the two lateral probe positions (i.e., left vs. middle-left and right vs. middle-right) did not differ between alerting conditions (both $p_{\mathrm{Holm}} \mathrm{s}>.99$ ). In contrast, the RT difference between the middle and middle-right probe position differed between trials with and without an alerting signal $\left(p_{\text {Holm }}=.008\right)$, and a trend in the same direction was observed for the comparison of the RT difference between the middle and middle-left position $\left(p_{\mathrm{Holm}} \mathrm{s}=.07\right)$.

\section{Discussion}

Experiment 1 revealed a sizeable performance benefit due to the alerting signal: Participants responded faster in trials with an alerting signal than in trials without an alerting signal, thus replicating the results of previous studies (e.g., Hackley \& Valle-Inclán, 1998; Keuss, 1972). Furthermore, performance in the probe task was clearly affected by the probe's position, as reflected in a quadratic trend: Participants' responses were fastest to probes presented at the middle position, where the relevant information (the target) was presented in main task trials, and slowed down toward lateral positions. This V- shaped RT function directly replicates the RT results reported by LaBerge (1983). Most importantly, probe RT was also affected by the presence of an alerting signal. First, like in the main task, responses to probes were overall faster if an alerting signal was presented beforehand. Second, the presentation of an alerting signal also modulated the shape of the probe-RT function. Crucially, however, its effect did not show up in flattened probe-RT function, as would have been expected if the alerting signal broadened the attentional beam. Rather, the modulation of the probe-RT function by the alerting signal was due to an additional quartic trend in the probe-RT function that interacted with thealerting signal. Basically, the presence of a quartic trend indicates a three-way folding of the mathematical function that describes the dependent variable. In combination with the quadratic trend, the interaction of the alerting signal with the quartic trend may be best described as a specifically large RT difference between probes at the middle position and probes at the positions directly next to it (i.e., middle-left and middle-right; see Fig. 2, lower panel). In line with this interpretation, a post-hoc comparison of the RT difference between each two neighboring probe position showed that the RT difference between the middle position and its neighboring positions was larger in trials with alerting signals.

Before this pattern of results can be discussed any further, another result must be considered first, that is, the accuracy level in no-go trials: Specifically, whereas La Berge (LaBerge, 1983) reported mean accuracy values of at about $90 \%$ in the main task and $97 \%$ in the probe task, accuracy in no-go trials was on average at about $73 \%$ in the present experiment. Furthermore, in the probe task, accuracy in no-go trials was also modulated by alerting signal and probe position. Specifically, participants conducted more no-go errors in trials with an alerting signal, and they conducted more no-go errors specifically at the middle probe position. This result clearly suggests that participants in Experiment 1 had a bias toward responding even if the probe was a no-go stimulus. Although it can only be speculated about the reasons for this elevated error rate, the most obvious difference between Experiment 1 and the LaBerge study is the presentation of an intense auditory stimulus as alerting signal in half of the trials, which may have increased the overall level of arousal (for evidence that alerting signals increase arousal, see, e.g., Posner, Klein, Summers, \& Buggie, 1973). In combination with the instruction to respond as fast as possible to go stimuli, this may have induced a bias toward responding, specifically at the middle probe position, because this position was occupied by taskrelevant stimuli in the majority of the trials.

With respect to the above-described probe-RT function, the drop in accuracy at the middle probe position may partially explain the observation of a quartic trend: As explicated above, this trend most likely reflects a specific RT benefit at the middle probe position, and especially in trials with an alerting signal. Given that this position also showed a drop 
in accuracy, it is possible that some part of the RT effect that is captured by the quartic trend origins from a bias towards responding. Importantly, however, it is unlikely that the effect on accuracy accounts for the quadratic trend in the probe-RT function: Specifically, the quadratic trend indexes a steady increase from the middle probe position to the outward probe positions (see also LaBerge, 1983). In line with this interpretation, a post-hoc analysis of the main effect of probe position showed that RT was shorter at the middle as compared to the middle-left and middle-right probe positions, and RT at these positions was shorter than at the most outward (i.e., left and right) probe positions. In contrast, accuracy in no-go probe trials showed a specific drop at the middle probe position, but did not differ between lateral positions, as would have been expected if it accounted for the quadratic trend. Hence, it seems save to conclude that the quadratic trend in the probeRT function captured the size of the attentional focus. Furthermore, it seems save to conclude that this trend was not modulated by the presence of an alerting signal. In sum, the results of Experiment 1 do not support the hypothesis that alerting signals increase the focus of spatial attention.

One potential criticism against this interpretation could be that participants' focus of attention in the main task was measured rather indirectly in Experiment 1. Specifically, main task and probe task in this experiment were presented in separate trials in order to keep the experimental setup as close as possible to the original setup used by LaBerge (1983, Exp. 1). This separation of main and probe task, however, also required that the alerting signal was presented in both the main and the probe task. Therefore, it could be argued that the alerting effect on the probe-RT function in Experiment 1 primarily reflects its effect on processing the probe, rather than a direct effect on the attentional focus in the main task. To address this potential criticism, a second experiment was conducted in which the size of the attentional focus in the main task was measured directly.

\section{Experiment 2}

Experiment 2 served as a replication and methodological refinement of Experiment 1. First of all, the size of the attentional focus in the main task was measured in a more direct manner, by presenting the probe task immediately after the main task. At the same time, the amount of additional resources required for processing the probe itself was reduced to a minimum. Therefore, the nature of the probe task was changed in the following two ways: First, instead of a letter or digit, the probe was now a small dot (see Fig. 1B). Second, participants did not perform a go-no-go response, but instead had to respond as soon as they detected the probe. As in Experiment 1, it was assumed that the target in the main task would center participants' attention at its position.
Accordingly, RT to probes presented directly after the main task should be fastest at that position and increase toward more lateral positions. This RT pattern should again result in a V-shaped probe-RT function. Finally, and most importantly, if the size of the attentional focus in the main task were increased by an alerting signal, this should be directly reflected in the shape of the probe-RT function: Specifically, in trials with an alerting signal, a flattened probe-RT function should be observed.

\section{Method}

Participants Fourty-two new participants were recruited for Experiment 2. Four participants had to be excluded, because three of them made excessive anticipations to probes (i.e., RT $<150 \mathrm{~ms}$ ) and one responded to no-go stimuli in the main task on more than $75 \%$ of the trials. The final sample consisted of 28 women and ten men $\left(M_{\text {age }}=24.13\right.$ years, age range: $19-47$ years). All participants had normal or corrected-to-normal visual acuity and received either course credit or payment $(€ 12)$. Furthermore, all participants gave written informed consent prior to the experiment.

Apparatus and stimuli The apparatus and stimuli were the same as in Experiment 1, except for the probe display, which now consisted of a small white dot (diameter: $0.2 \times 0.2^{\circ}$ of visual angle; see Fig. 1B).

Procedure The procedure was identical to that of Experiment 1, except for the following changes: First, the probe-task display was now presented immediately after participants had responded to the main task. Second, the ratio of main-toprobe task trials, and the ratio of go to no-go trials in the main task was changed to $2: 1$ and $3: 1$, respectively, in order to increase the number of trials left for RT analysis. Participants now performed 720 experimental trials in total, which were presented in two blocks of 360 trials each. A break was inserted after each 36 trials. Within each block, 240 trials consisted of main task trials only, with 180 trials of them being go trials, and 60 trials being no-go trials. The remaining 120 trials within each block consisted of the main task plus the probe task. The main task in these main + probe task trials required a go response in 90 trials (go trials), but no response in 30 trials (no-go trials). Finally, due to the change in the ratio of go and no-go trials, each practice block now contained 12 trials. Overall, an experimental session lasted at about $70 \mathrm{~min}$.

\section{Results}

As in Experiment 1, practice trials were excluded from data analysis. Furthermore, for analysis of RT, trials with RTs below $150 \mathrm{~ms}$ or above $1,400 \mathrm{~ms}$ were excluded $(M=0.24 \%$ of the trials in the main task, and $M=0.08 \%$ of the trials in the 
probe task). Mean accuracy in the probe task was very high ( $M$ $=94.6 \%)$ and close to ceiling in go trials of the main task $(M=$ 99.7\%), so that accuracy in these conditions was not further analyzed. In contrast, mean accuracy in the no-go trials of the main task was $M=84.7 \%$ and was therefore analyzed.

For statistical analysis of the main task, a one-way rmANOVA with the factor alerting signal (with alerting signal, without alerting signal) was conducted on mean RT for correct responses in go trials and mean accuracy in no-go trials. For statistical analysis of the probe task, a two-way rmANOVA with the factor alerting signal (with alerting signal, without alerting signal) and probe position (left, middle-left, middle, middle-right, right) was conducted on mean RT for correct responses. Analogously to Experiment 1, a trend analysis was conducted on the standardized probe RT in order to model the shape of the probe-RT function. Mean RT in the main and the probe task is depicted in Fig. 3.

As in Experiment 1, mean RT in the main task was shorter in trials with an alerting signal than in trials without an alerting signal, $F(1,37)=106.64, p<.001, \eta_{\mathrm{p}}{ }^{2}=.74$ (see Fig. 3, upper panel). Furthermore, accuracy in no-go trials in the main task did not differ between trials with an alerting signal $(M=$ $85.4 \%)$ and trials without an alerting signal $(M=84.1 \%)$, $F(1,37)=1.72, p=.20$. Analysis of the mean RTs in the probe task revealed a main effect of probe position, $F(1,37)=8.75$, $p<.001, \eta_{\mathrm{p}}{ }^{2}=.19$, since RT clearly varied as a function of the probe's position (see Fig. 3, lower panel). There was no main effect of alerting signal on probe RT, $F(1,37)=0.91, p=.35$, but an interaction of probe position and alerting signal, $F(4$, 148) $=2.91, p=.024, \eta_{\mathrm{p}}{ }^{2}=.07$.

The trend analysis of standardized probe RT revealed a quadratic trend for the effect of probe position, $F(1,37)=$ $32.0, p<.001, \eta_{\mathrm{p}}{ }^{2}=.46$. Importantly, this quadratic trend was not modulated by alerting signal, $F(1,37)=1.70, p=$ .20. Instead, the effect of the alerting signal was reflected in an additional quartic trend, $F(1,37)=6.07, p=.02, \eta_{\mathrm{p}}{ }^{2}=.14$. No further meaningful trends or modulations of these trends by alerting signal were observed (all $F_{\mathrm{s}}<3.21$ ).

\section{Discussion}

Experiment 2 replicated several basic findings of Experiment 1: First, a sizable alerting effect was observed in the main task, as participants responded faster in trials with an alerting signal than in trials without an alerting signal. Second, detection of the probe was again fastest at the middle probe position and slowed down toward more lateral positions, as reflected in the main effect of probe position and the quadratic trend in the probe-RT function. Hence, the main task in Experiment 2 successfully induced a focus of attention that was centered at the middle display position. Furthermore, like in Experiment 1, the probe-RT function was also modulated by the presence of the alerting signal, as reflected in the interaction of alerting

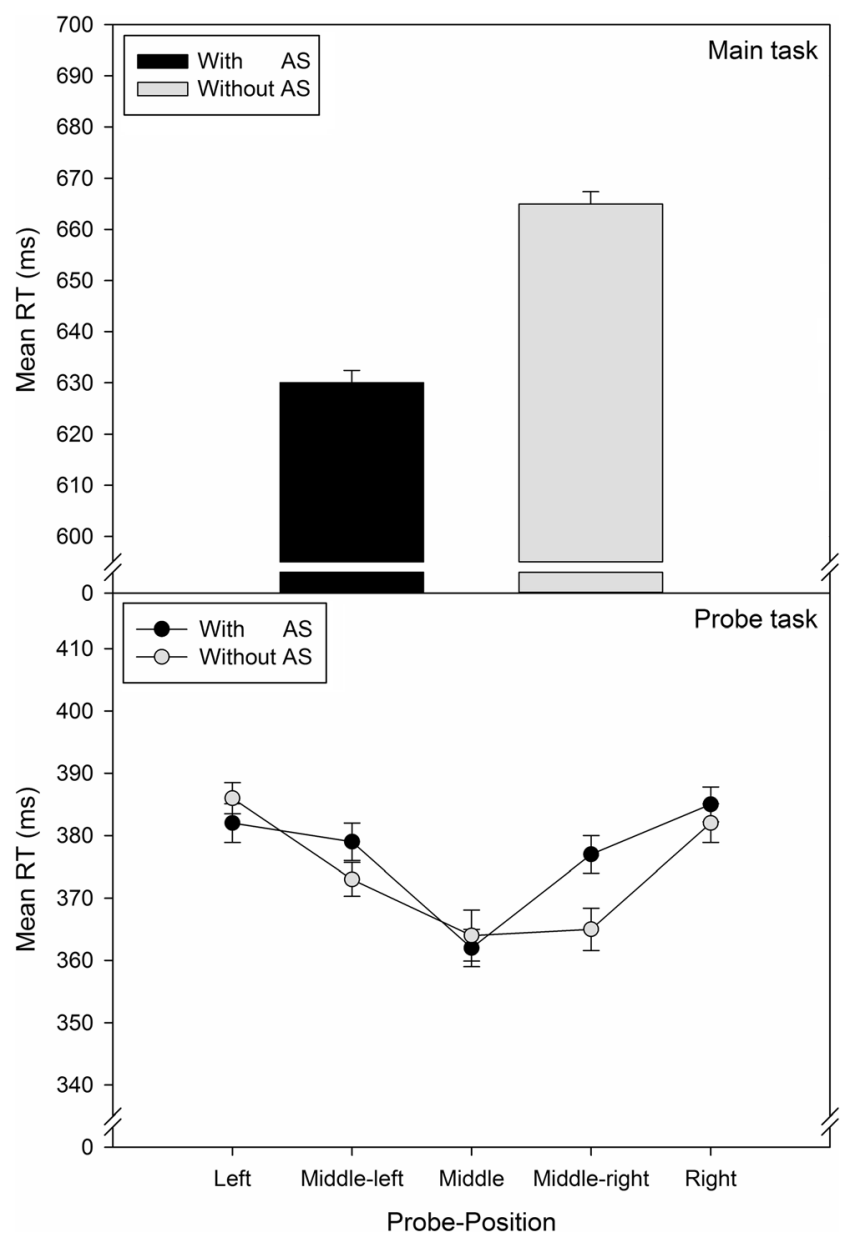

Fig. 3 Results of Experiment 2. (Upper panel) Mean reaction time (RT) in the main task as a function of alerting signal (AS). (Lower panel) Mean $\mathrm{RT}$ in the probe task as a function of AS and probe position.

signal and probe position. Importantly, however, the trend analysis in Experiment 2 again showed that alerting signals did not modulate the quadratic trend in the probe-RT function, indicative of the size of the attentional focus (LaBerge, 1983). Instead, alerting signals led to an additional quartic trend in the probe-RT function. Unlike in Experiment 1, however, this observation cannot be ascribed to a specifically large alerting effect at the middle probe position, because there was no overall effect of the alerting signal on probe RT. Rather, this trend reflects an additional folding of the probe-RT function at the middle-left and middle-right positions in trials with an alerting signal, which was absent in trials without an alerting signal (see Fig. 3, lower panel).

In sum, the results of Experiment 2 show that presenting the probe task directly after the main task successfully captured the attentional focus that was centered at the middle display position. Yet, Experiment 2 did not reveal a flattening of the probe-RT function in trials with an alerting signal, which is again inconsistent with the idea of an increased focus of spatial attention being caused by alerting signals. 


\section{Experiment 3}

The experiments reported so far do not provide any support for the notion that alerting signals increase the focus of spatial attention and thereby lead to larger congruency effects. Nevertheless, before drawing any decisive conclusion, it must be considered that the hypothesis of a larger attentional focus was originally formulated in the context of a flanker task in which there was a conflict between the flankers and the target (Weinbach \& Henik, 2012, Exp. 4). In comparison to this task, the experimental setup of the present study differed in two important ways: First, the flankers in Experiments 1 and 2 were always neutral, in the sense that they did not have any direct association with a target response. Second, the flankers were letters, whereas the flankers in the previous studies were arrows (e.g., Weinbach \& Henik, 2012). Importantly, some studies have shown that arrow-like stimuli lead to an automatic shift of attention in their intended direction (see, e.g., Ristic, Friesen, \& Kingstone, 2002; Tipples, 2002), and may even activate corresponding spatial responses - as reflected, for instance, by an LRP response in the event-related potential (Eimer, 1995; Willemssen, Hoormann, Hohnsbein, \& Falkenstein, 2004). Therefore, it is possible that alerting effects on visuospatial attention only emerge when spatial events (which may activate corresponding response codes) are presented in the attended visual field.

To examine this possibility, a third experiment was conducted in which the main task was modified in two ways: First, the letter flankers were replaced by arrows, which pointed to either the left or right. Second, the go-no-go response in the main task was replaced by a spatial choice response. Specifically, participants now responded to one set of target letters with a left keypress and to the other with a right keypress. Therefore, the irrelevant spatial information conveyed by the flankers (i.e., left or right) would either match or mismatch the spatial response, thus yielding congruent and incongruent conditions.

In analogy to Experiments 1 and 2, I expected that RT in the main task would overall be faster in trials with an alerting signal. Furthermore, as was reported by Weinbach and Henik (2012, Exp. 4), the use of arrow flankers should lead to a congruency effect, and this effect should be more pronounced in trials with an alerting signal. Most importantly, if the alerting effect on spatial attention depends on the presence of spatial information, Experiment 3 should now reveal the modulation of the probe-RT function: Specifically, it should be flattened in trials with an alerting signal, as compared to trials without an alerting signal.

\section{Method}

Participants Fourty new participants were recruited for Experiment 3. Two participants had to be excluded because of excessive anticipatory responses to probes (i.e., RT $<150$ $\mathrm{ms})$. The final sample consisted of 31 women and seven men $\left(M_{\text {age }}=22.08\right.$ years, age range: $18-29$ years $)$, who had normal or corrected-to-normal visual acuity and received either course credit or payment $(€ 12)$. All participants gave written informed consent prior to their participation.

Apparatus and stimuli The apparatus and stimuli were identical to those of Experiment 2, except that the flankers now were arrows, which pointed to either the left or right (see Fig. 1C). The arrows had the same size as the central target letter (height $\times$ width: $0.5 \times 0.5^{\circ}$ of visual angle) and an edge thickness of $0.16^{\circ}$ of visual angle. To avoid confusion between responding to the target and the probe, responses in the two tasks were executed with different hands. Specifically, the responses in the main task were executed with the right hand, using a custom-made response box, whereas the responses in the probe task were executed with the left hand, using the space bar of a standard keyboard. To ensure that participants did not use a spatial response mapping for the probe response, the keyboard was positioned in such a manner that the space bar was located directly in front of participants and roughly in front of the center of the screen.

Procedure The procedure was identical to that of Experiment 2, except for the following changes: First, the main task in Experiment 3 required a choice response. Specifically, depending on the target letter set (i.e., A, B, C, D, and E vs. N, $\mathrm{O}, \mathrm{P}, \mathrm{R}$, and $\mathrm{S}$ ), participants responded with keys positioned below their right index and middle fingers. To stress the spatial nature of the main task, the responses were explicitly designated as "left response" and "right response" in the instructions. In contrast, the response instructions for the probe task did not contain any explicit spatial reference; instead, participants were simply instructed to use the space bar. To emphasize correct responding in both tasks, participants received error feedback in the main task whenever they responded with the wrong key or too slowly (i.e., RT > 1,500 ms); similarly, participants received error feedback in the probe task whenever they made an anticipatory response (i.e., RT $<150 \mathrm{~ms}$ ) or did not respond at all (i.e., RT $>1,500 \mathrm{~ms}$ ). The assignment of left and right keys to the two target sets in the main task was counterbalanced across participants.

\section{Results}

Practice trials were discarded from the data analysis. Again, trials with RT below $150 \mathrm{~ms}$ or above $1,400 \mathrm{~ms}$ were excluded from the RT analysis $(M=0.21 \%$ in the main task and $M=$ $0.02 \%$ in the probe task). Overall, accuracy was high for both the main task $(M=95.9 \%)$ and the probe task $(M=95.4 \%)$, but was not at ceiling, so that error rates could be analyzed. 
For the analysis of performance in the main task, separate two-way rmANOVAs with the factors alerting signal (with alerting signal, without alerting signal) and congruency (congruent, incongruent) were performed on the mean RTs for correct responses and on mean accuracy. Similarly, for the analysis of performance in the probe task, separate two-way rmANOVAs with the factors alerting signal (with alerting signal, without alerting signal) and probe position (left, middleleft, middle, middle-right, right) were conducted on mean RT for correct responses and on mean accuracy. Again, a trend analysis was performed on the standardized probe RT. Mean $\mathrm{RT}$ in the main and the probe task is depicted in Fig. 4.

For the main task, the analysis of mean RT revealed a main effect of alerting signal, $F(1,37)=344.24, p<.001, \eta_{\mathrm{p}}{ }^{2}=.90$, as well as a main effect of congruency, $F(1,37)=54.91, p<$ $.001, \eta_{\mathrm{p}}{ }^{2}=.60$. Participants responded faster in trials with an alerting signal than in trials without an alerting signal, and they responded faster in congruent than in incongruent trials (see Fig. 4, upper panel). Unexpectedly, however, no interaction of alerting signal and congruency emerged, $F(1,37)=$ $0.08, p=.78$. The analysis of mean accuracy in the main task revealed only a main effect of congruency, $F(1,37)=40.08, p$ $<.001, \eta_{\mathrm{p}}{ }^{2}=.52$, since participants responded more accurately in congruent $(M=97.0 \%)$ than in incongruent $(M=94.8 \%)$ trials. No main effect of alerting signal or interaction with congruency was observed on mean accuracy (both $F \mathrm{~s}<1.8$ ). For the probe task, the analysis of mean RT revealed a main effect of probe position, $F(4,148)=13.80, p<.001, \eta_{\mathrm{p}}{ }^{2}=.27$, since RT was fastest at the middle probe position and increased toward more lateral positions (see Fig. 4, lower panel). Furthermore, there was also a small but reliable main effect of alerting signal, $F(1,37)=4.57, p=.04, \eta_{\mathrm{p}}{ }^{2}=.11$, reflecting faster RT in trials with an alerting signal than in trials without an alerting signal. No interaction between the two factors was observed, $F(1,37)=0.16, p=.92$. The analysis of mean accuracy in the probe task did not reveal any effects at all (all $F \mathrm{~s}<1.3)$.

As in the previous experiments, the trend analysis on standardized probe RT revealed a prominent quadratic trend for the effect of probe position, $F(1,37)=38.41, p<.001, \eta_{\mathrm{p}}{ }^{2}=$ .51. Furthermore, the effect of probe position was also reflected in a quartic trend, $F(1,37)=24.63, p<.001, \eta_{\mathrm{p}}{ }^{2}=$ .40 , since probe RT did not increase toward lateral positions in a strictly linear way, but leveled off toward the most outward positions (see Fig. 4, lower panel). Importantly, these two trends were not modulated by the alerting signal (both $F_{\mathrm{s}}<$ 1 ), nor were there any further trends or modulations of these trends by alerting signal (all $F_{\mathrm{S}}<1$ ).

\section{Discussion}

Experiment 3 replicated several important findings from the previous experiments. First of all, an overall response benefit

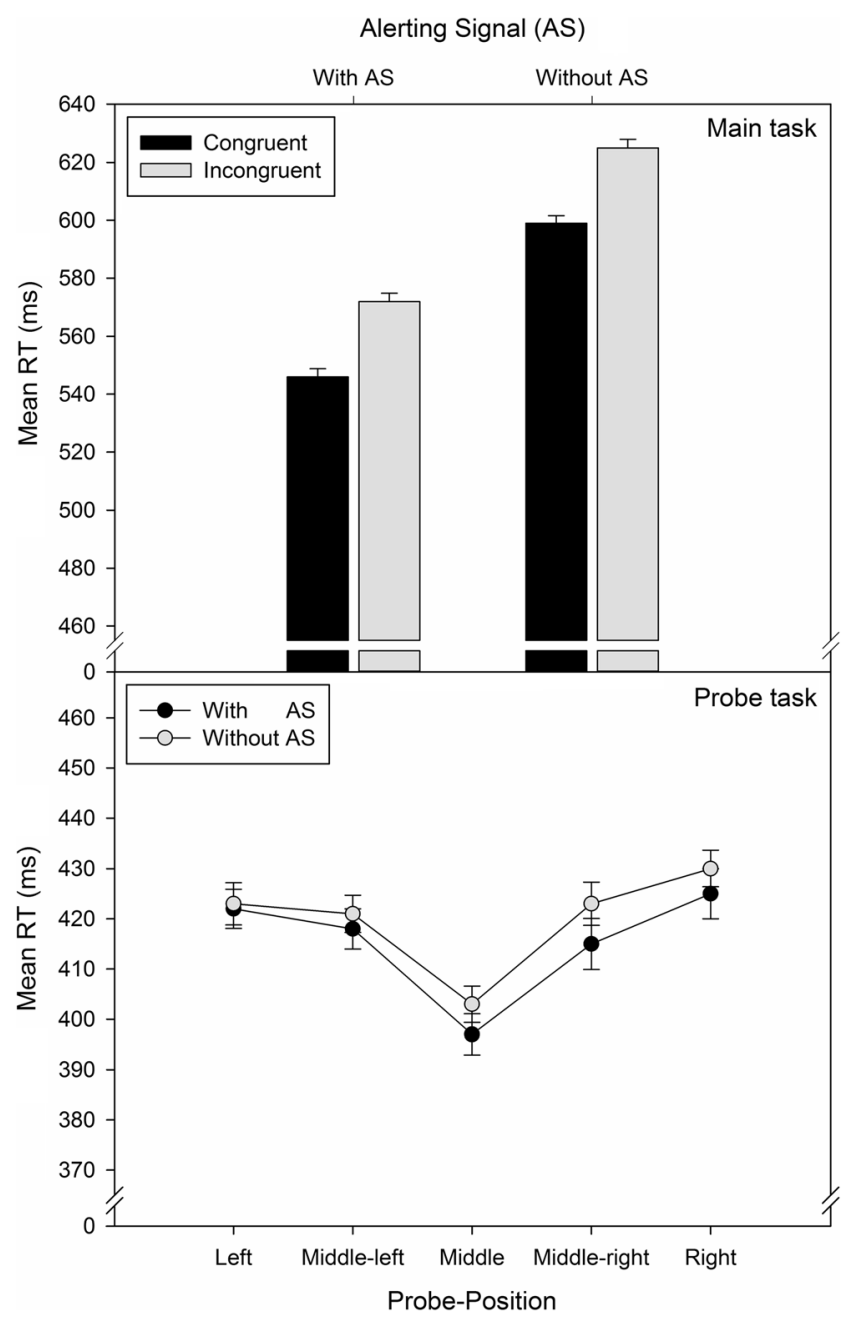

Fig. 4 Results of Experiment 3. (Upper panel) Mean reaction time (RT) in the main task as a function of alerting signal (AS) and congruency. (Lower panel) Mean RT in the probe task as a function of AS and probe position.

due to the alerting signal was observed in the main and probe task, as participants responded faster in trials with an alerting signal than in trials without an alerting signal. Second, as in Experiments 1 and 2, the RT in the probe task varied as a function of the probe's position, as reflected in the main effect of probe position and in a pronounced quadratic trend. Importantly, however, the quadratic trend, indicative of the size of the attentional focus, was not modulated by the presence of the alerting signal. This result is again at variance with the idea that alerting signals increase the size of the attentional focus.

Apart from a replication of the major findings in Experiments 1 and 2, Experiment 3 also revealed a congruency effect in the main task: Specifically, participants responded more slowly and less accurately when the target letter was flanked by arrows that pointed in the direction opposite to the required response. Therefore, Experiment 3 successfully replicated Weinbach and Henik's (2012, Exp. 4) result and shows 
that arrow flankers can induce congruency effects, even if they are never presented as targets and thus are completely taskirrelevant. The observation of a congruency effect in this specific context suggests that the spatial information conveyed by arrows is processed automatically to a certain extent, and thereby affects responding to another unrelated stimulus. In contrast to the results of Weinbach and Henik (2012, Exp. 4), however, the alerting signal in Experiment 3 did not modulate the size of the congruency effect. Since the observation of such an interaction is crucial for the interpretation of an alerting effect on probe RT, a fourth experiment was conducted.

\section{Experiment 4}

Experiment 4 served as a direct replication of Experiment 3, with the aim of increasing the likelihood of observing a congruency-by-alerting interaction in the main task. Specifically, one major difference between Weinbach and Henik's (2012) study and Experiment 3 of the present study was the use of a larger set size of targets per response category: Whereas the participants in Weinbach and Henik's (2012) study had to monitor only one target per response category (i.e., the color green vs. red), the participants in Experiment 3 had to monitor five different targets per response category (i.e., the letters A, B, C, D, and E vs. the letters N, O, P, R, and S). Furthermore, the targets in Weinbach and Henik's (2012) study were simple patches, whereas the targets in Experiment 3 were letters, and thus more different from each other in their basic features. It seems reasonable to assume that the overall processing requirements in the latter situation would be much higher and therefore prolong overall processing time. Importantly, this might prevent the alerting signal from affecting response selection, either because the activation of irrelevant spatial codes might already have faded out when response selection took place or because more controlled stimulus processing might lead to better shielding against the irrelevant spatial code. In support of the former idea, Miller (1991, Exp. 7) reported that the congruency effect in a classical letter flanker task was largely reduced if the target was embedded in a visual search display, and the decision about its identity was thereby rendered more difficult. Miller reasoned that presenting a target together with additional distractors increases the overall processing load and thereby delays responding (Miller, 1991, p. 285). This delay in responding would then diminish the impact of the flankers. Similarly, a delay in responding due to a large target set size might have reduced the probability of observing a congruency-by-alerting interaction in Experiment 3. Such an account in terms of relative timing differences of the alerting effect and the congruency effect seems plausible, given that irrelevant spatial information seems to be activated only temporarily (see, e.g., Hommel, 1993, for evidence in this regard in the context of the Simon task).

On grounds of this possibility, the experimental setup was changed in such a way that the overall processing time regarding the target would be reduced: First, the target set size for each category was reduced from five letters to only one. Second, the letters A and B were chosen as the targets, to ensure that they would be easily discriminable on grounds of their basic visual features. The reduction of the target set size and the choice of easily discriminable letters should reduce the processing requirements and therefore leave some space for the alerting signal to increase the impact of the arrow flankers. Accordingly, I expected that Experiment 4 would reveal the expected congruency-by-alerting interaction in the main task.

\section{Method}

Participants A sample of thirty-eight new participants (34 women, four men; $M_{\text {age }}=23.7$ years, age range: $19-41$ years) took part in Experiment 4. All of them had normal or corrected-to-normal visual acuity and received either course credit or payment $(€ 12)$; furthermore, all participants gave written informed consent prior to the experiment.

Apparatus and stimuli The apparatus and stimuli were identical to those of Experiment 3, except that only the letters A and $\mathrm{B}$ were presented as the central target letters.

Procedure The procedure was identical to that of Experiment 3 , except that participants now responded with a left keypress only to one target letter (e.g., the letter A), and with a right keypress to the other target letter (e.g., the letter B). The assignment of left and right responses to the two target letters was counterbalanced across participants.

\section{Results}

Practice trials were excluded from the data analysis. Furthermore, for the RT analysis, trials with RT below $150 \mathrm{~ms}$ or above $1,400 \mathrm{~ms}$ were excluded ( $M=0.07 \%$ in the main task and $M=0.4 \%$ in the probe task). Mean accuracy in both tasks was high (i.e., $M=97.2 \%$ in the main task and $M=$ $96.9 \%$ in the probe task), but not at ceiling, and thus could be analyzed.

Statistical analyses were conducted analogously to those in Experiment 3: For the main task, separate two-way rmANOVAs with the factors alerting signal (with alerting signal, without alerting signal) and congruency (congruent, incongruent) were conducted on the mean RT for correct responses and on mean accuracy. For the probe task, separate two-way rmANOVAs with the factors alerting signal (with alerting signal, without alerting signal) and probe position (left, middle-left, middle, middle-right, right) were conducted 
on the mean RT for correct responses and on mean accuracy. Again, a trend analysis (using orthogonal polynomial contrasts) with the same factors was conducted on the standardized probe RT. The results are depicted in Fig. 5.

The analysis of mean RT in the main task revealed main effects of both alerting signal, $F(1,37)=275.36, p<.001, \eta_{\mathrm{p}}{ }^{2}$ $=.88$, and congruency, $F(1,37)=45.64, p<.001, \eta_{\mathrm{p}}{ }^{2}=.55$. Participants responded faster in trials with an alerting signal than in trials without an alerting signal, and they responded faster in congruent than in incongruent trials (see Fig. 5, upper panel). Most importantly, the size of the congruency effect was modulated by alerting signal, $F(1,37)=4.48, p=.04, \eta_{\mathrm{p}}{ }^{2}=$ .11. Specifically, the congruency effect $\left(\mathrm{RT}_{\text {incon }}\right.$ minus $\left.\mathrm{RT}_{\text {con }}\right)$ was larger in trials with an alerting signal $(M=36 \mathrm{~ms})$ than in trials without an alerting signal $(M=28 \mathrm{~ms})$. The analysis of mean accuracy in the main task revealed no main effect of alerting signal, $F(1,37)=2.58, p=.12$, but a main effect of congruency, $F(1,37)=26.53, p<.001, \eta_{\mathrm{p}}{ }^{2}=.42$, since mean accuracy was higher in congruent $(M=98.4 \%)$ than in incongruent $(M=96.0 \%)$ trials. Furthermore, there was an interaction of the two factors, $F(1,37)=14.57, p<.001, \eta_{\mathrm{p}}{ }^{2}=.28$, since the difference in accuracy between congruent and incongruent trials was larger in trials with an alerting signal $(M=$ $3.1 \%)$ than in trials without an alerting signal $(M=1.6 \%)$. For the probe task, the analysis of mean RT revealed only a main effect of probe position, $F(4,148)=13.08, p<.001, \eta_{\mathrm{p}}{ }^{2}=.26$, but no main effect of alerting signal or interaction between the two factors (both $F \mathrm{~s}<1$ ). Finally, the analysis of mean accuracy in the probe task revealed no main effects (both $F_{\mathrm{S}}<1.7$ ), only a trend toward an interaction of probe position and alerting signal $F(4,148)=2.47, p=.06, \eta_{\mathrm{p}}{ }^{2}=.06$.

The trend analysis on standardized probe RT again revealed a pronounced quadratic trend for the effect of probe position, $F(1,37)=21.98, p<.001, \eta_{\mathrm{p}}{ }^{2}=.37$, as reflected in a V-shaped RT function (see Fig. 5, lower panel). Importantly, this quadratic trend was not modulated by alerting signal, $F(1,37)=$ $1.27, p=.27$. Apart from the quadratic trend, the effect of probe position was also reflected in a quartic trend, $F(1,37)$ $=13.54, p=.001, \eta_{\mathrm{p}}{ }^{2}=.27$. Finally, the effect of probe position was reflected in a marginally significant linear trend, $F(1$, $37)=4.02, p=.05, \eta_{\mathrm{p}}{ }^{2}=.10$. There were no further trends for the effect of probe position, nor modulations of these trends by alerting signal (all $F_{\mathrm{S}}<1.8$ ).

\section{Discussion}

Experiment 4 revealed several important findings: First, and most relevant, the expected congruency-by-alerting interaction was now observed in the main task. Participants were not only overall faster in trials with an alerting signal, but also showed a larger congruency effect, and this effect was present in both RTs and error rates. The observation of a larger congruency effect in trials with an alerting signal directly

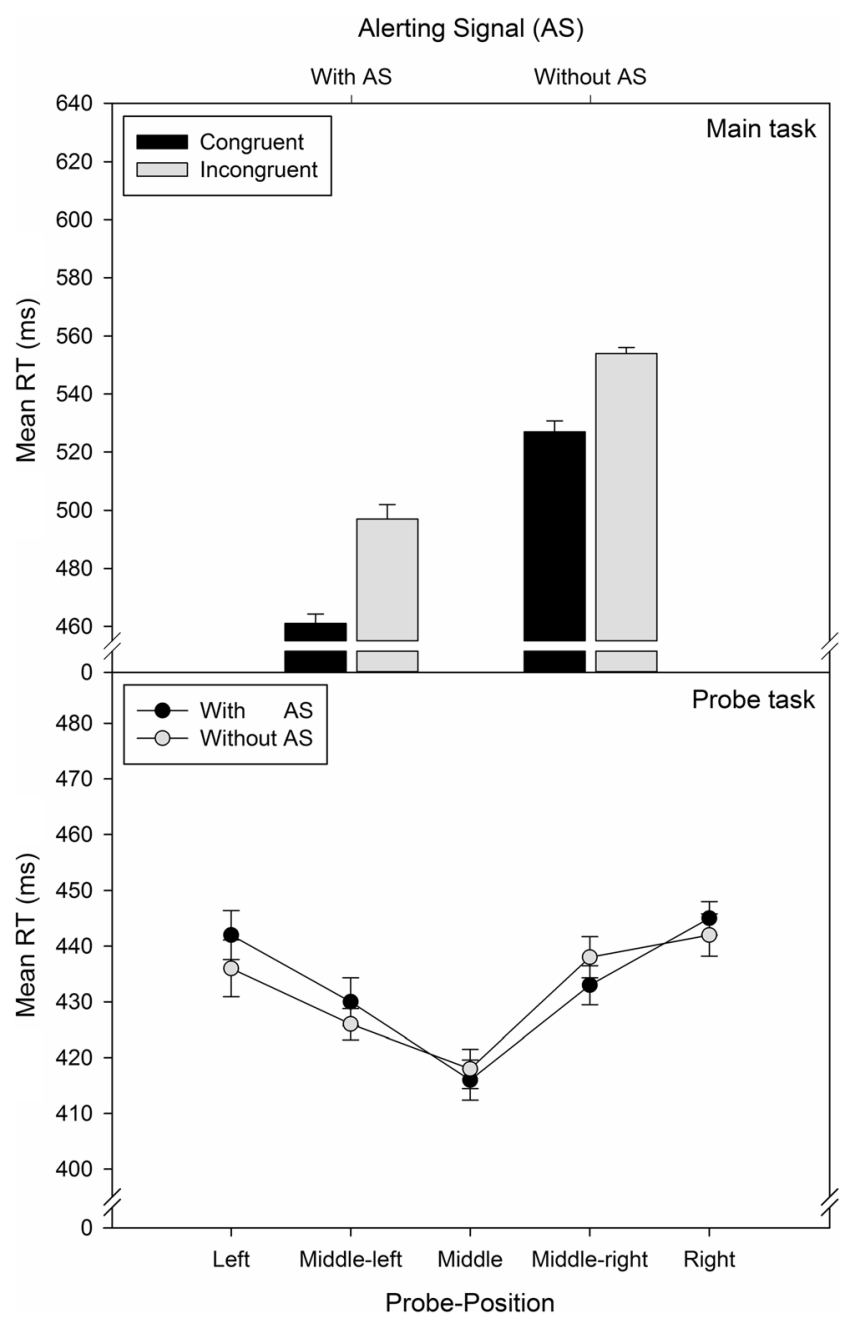

Fig. 5 Results of Experiment 4. (Upper panel) Mean reaction time (RT) in the main task as a function of alerting signal (AS) and congruency. (Lower panel) Mean RT in the probe task as a function of AS and probe position.

replicates the results of Weinbach and Henik (2012, Exp. 4), who were the first to show that alerting signals increase conflict in the flanker task even if the flankers are not drawn from the same stimulus set as the target, but only share a spatial feature with the target response. Furthermore, it shows that the modification of the experimental setup from Experiment 3 to Experiment 4 was effective in revealing the expected congruency-by-alerting interaction.

As was mentioned before, the crucial difference between Experiments 3 and 4 was a reduction in the number of targets that had to be mapped to one of the two response categories. Whereas the participants in Experiment 3 had to map five different letters onto each response category, the participants in Experiment 4 had a one-to-one mapping of letters to response categories. This modification was made on grounds of the assumption that a larger target set size might increase the overall processing requirements, since a larger number of items would have to be kept in working memory and have to 
be translated into a corresponding response. In the Discussion of Experiment 3, I reasoned that an increase in processing requirements might lead to a stronger temporal dissociation of the alerting effect and the process of response selection, which would give rise to the congruency effect. Such a scenario rests upon the assumption that the alerting effect on the flankers is rather short-lived and will already have faded out if response selection starts relatively late, as might be the case in the context of a large target set size. An alternative scenario is that participants might adopt a more restrictive processing mode to handle the large target set. Specifically, participants might either narrow down their attentional focus or concentrate more processing resources at the target position. ${ }^{1}$ This restrictive processing mode would then shield target processing against the impact of the flankers and thereby prevent the emergence of a congruency-by-alerting interaction.

To gain some empirical evidence on these two scenarios, I conducted two additional analyses: The first analysis comprised a direct between-experiments comparison of mean RTs in the main task, to investigate whether the alerting effects differed between Experiments 3 and $4 .^{2}$ Specifically, if the assumption of a stronger temporal dissociation of the alerting effect and response selection is correct, the alerting effect should be weaker in Experiment 3 than in Experiment 4. In contrast, if the assumption of a narrower focus of attention is correct, the impact of the flankers, and thus the congruency effect, should be overall weaker in Experiment 3 than in Experiment 4 (see, e.g., LaBerge \& Brown, 1986). The second analysis comprised a direct comparison of the shape of the

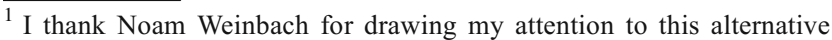
possibility.

${ }^{2}$ Specifically, I conducted a mixed ANOVA on mean RT in the probe task, with the within-subjects factors alerting signal (with alerting signal, without alerting signal) and congruency (congruent, incongruent) and the betweensubjects factor experiment (Exp. 3, Exp. 4). This analysis revealed main effects of congruency, $F(1,74)=96.78, p<.001, \eta_{\mathrm{p}}{ }^{2}=.57$, alerting signal, $F(1,74)=$ $599.02, p<.001, \eta_{\mathrm{p}}{ }^{2}=.89$, and experiment, $F(1,74)=29.70, p<.001, \eta_{\mathrm{p}}{ }^{2}=$ .29. The latter main effect was due to overall longer RT in Experiment 3 ( $M=$ $586 \mathrm{~ms})$ than in Experiment $4(M=510 \mathrm{~ms})$. Furthermore, this analysis revealed a marginally significant interaction of alerting signal with experiment, $F(1,74)=3.19, p=.08, \eta_{\mathrm{p}}{ }^{2}=.04$, which reflected a smaller alerting effect in Experiment $3(M=53 \mathrm{~ms})$ than in Experiment $4(M=62 \mathrm{~ms})$. There was no congruency-by-experiment interaction, $F(1,74)=1.06, p=.31$, nor a threeway interaction of congruency, alerting signal, and experiment, $F(1,74)=$ $2.64, p=.11$. Instead, there was a marginally significant interaction of congruency with alerting signal, $F(1,74)=3.70, p=.06, \eta_{\mathrm{p}}^{2}=.05$.

${ }^{3}$ To this end, I conducted a trend analysis on the standardized probe RT, which included the within-subjects factors alerting signal (with alerting signal, without alerting signal) and probe position (left, middle-left, middle, middle-right, right), as well as the between-subjects factor experiment (Exp. 3, Exp. 4). Importantly, this analysis again revealed a pronounced quadratic trend, $F(1$, $74)=54.19, p<.001, \eta_{\mathrm{p}}{ }^{2}=.42$, and a quartic trend, $F(1,74)=38.06, p<.001$, $\eta_{\mathrm{p}}{ }^{2}=.34$, for the effect of probe position. Furthermore, a marginally significant linear trend emerged for the effect of probe position, $F(1,74)=3.81, p=.06$, $\eta_{\mathrm{p}}{ }^{2}=.05$. Most importantly, there was no difference between the experiments, regarding the quadratic trend, $F(1,74)=0.15, p=.70$, the quartic trend, $F(1$, $74)=2.17, p=.15$, or any other trend (all $\left.F_{\mathrm{S}}<2.1\right)$. Finally, none of the trends was modulated either by alerting signal (all $F_{\mathrm{S}}<1.3$ ) or by the interaction of alerting signal with experiment (all $F \mathrm{~s}<1$ ).
}

probe-RT functions in the two experiments. ${ }^{3}$ This analysis was conducted on the grounds of the assumption that stronger focusing of attention will also affect the slope of the quadratic trend in the probe-RT function (LaBerge, 1983; LaBerge \& Brown, 1986). Specifically, if attention was narrowed down onto the target in Experiment 3 in a stronger manner than in Experiment 4, the slope of the quadratic trend should be steeper in Experiment 3 than in Experiment 4. In short, the two analyses revealed that neither the congruency effects in the main task nor the slopes of the probe-RT functions differed between Experiments 3 and 4. Instead, these analyses indicated that the alerting effect on RTs was smaller in Experiment 3 than in Experiment 4, although this difference was only marginally significant. Taken together, this pattern of results seems to be more consistent with a scenario that assumes a temporal dissociation of the alerting effect and response selection than with a scenario that assumes stronger attentional focusing in Experiment 3.

Of course, the conclusions that can be drawn from these additional analyses are limited, given that they included a between-subjects comparison, which clearly has a lower power than a within-subjects comparison. Nonetheless, the observation that variations in the target set size can affect the impact of alerting signals on the congruency effect is interesting, because it complements previous results on potential boundary conditions for the observation of a congruency-by-alerting interaction. Specifically, as I explicated in the introduction, Weinbach and Henik (2012) showed that the spatial separation of relevant and irrelevant information plays a role in the emergence of the congruency-by-alerting interaction. Here, the results of Experiments 3 and 4 indicated that variations in processing requirements (such as the number of targets that have to be mapped to a response) may also be important: If they are rather low, the alerting signal will affect response selection, as reflected in increased congruency effects. If they are rather high, the alerting signal will not affect response selection. A stronger consideration of these processing requirements might thus be another interesting starting point when examining the specific conditions that give rise to the congruency-by-alerting interaction.

Apart from the observation of a congruency-by-alerting interaction, Experiment 4 also revealed the expected modulation of probe RT as a function of the probe's position: As in the previous experiments, the probe-RT function exhibited a clear $\mathrm{V}$ shape, indicative of the attentional focus. Hence, the main task in Experiment 4 was again effective in inducing a narrow focus of attention centered at the middle probe position. Furthermore, the probe-RT function was again characterized by a quartic trend, reflecting an additional folding of the probe-RT function. Despite the clear effect of the probe's position on RTs, Experiment 4 did not reveal supporting evidence for a modulation of the attentional focus by alerting signals: Specifically, the alerting signal did not affect the 
quadratic trend in the probe-RT function, indicative of the attentional focus. Hence, Experiment 4 again does not seem to support the idea of a larger attentional focus underlying the congruency-by-alerting interaction.

The validity of this statement, however, is disputable if one considers another aspect of the results: Specifically, and in contrast to the previous experiments, the alerting signal in Experiment 4 did not modulate RT in the probe task at all, in either the analysis of mean RT or the trend analysis of standardized probe RT. Although it is unclear why the alerting signal did not affect probe RTs in Experiment 4 at all, one potential factor that might have contributed to this null result could be that the probe directly followed the main task. As was explicated before, this trial procedure was chosen to capture directly the effect that the alerting signal exerted on the main task. However, this procedure has two potential side effects: First, it is possible that the specific effects in the main task would directly transfer to the probe task. For instance, the experience of response conflict in incongruent trials in the main task might lead participants to adopt a more conservative response criterion, and this strategy change might then absorb a potential alerting effect on probe RT. Second, because the probe task always followed the main task, the stimulus onset asynchrony (SOA) between the alerting signal and the probe task was rather long. Crucially, however, it has been shown that the arousing property of alerting signals may be strongest at short SOAs (see, e.g., Bertelson \& Tisseyre, 1969; Keuss, 1972). Therefore, the rather long SOA in Experiment 4 might have prevented the observation of an alerting effect on the quadratic trend in the probe-RT function.

In summary, Experiment 4 successfully replicated the expected congruency-by-alerting interaction, observed in previous studies (e.g., Weinbach \& Henik, 2012). Furthermore, Experiment 4 again did not show a flattening of the probeRT function, as would have been expected if alerting signals widened the attentional focus. Yet, the lack of an overall alerting effect on RT in the probe task limits the conclusions that can be drawn from Experiment 4. Therefore, a fifth experiment was conducted.

\section{Experiment 5}

Given that the experimental setup of Experiment 4 might have been ineffective in measuring an alerting effect on the attentional focus - because of either some transfer effect or the long SOAthe main aim of Experiment 5 was to replicate Experiment 4, but to ensure that the alerting signal would be effective in modulating processing of the probe. To this end, I drew on the basic trial procedure used in Experiment 1: Specifically, the probe task was decoupled from the main task, so that both tasks were now presented in separate trials. At the same time, the alerting effect in the probe task was now measured by presenting an alerting signal also in half of the probe-task trials. In light of Experiment 1's results, I expected that this experimental setup would allow for measuring a pronounced alerting effect in the probe task, while minimizing potential transfer effects from the main task.

A second modification in Experiment 5 concerned the nature of the main task: Specifically, although Experiment 4 replicated the observation of a congruency-by-alerting interaction, the size of this interaction was seemingly smaller than the size of the interactions reported in previous studies. For instance, in Experiment 4 of Weinbach and Henik's (2012) study, which is closest to Experiment 4 of the present study in terms of the task setup, the congruency effect was on average about $21 \mathrm{~ms}$ larger on trials with an alerting signal, whereas it was only about $8 \mathrm{~ms}$ larger in Experiment 4 of the present study. Hence, it is possible that the alerting-by-congruency interaction in Experiment 4 was not at its maximum, and that a modulation of the size of the attentional focus might occur only in case of a more dramatic interaction. To account for this possibility, the main task of Experiment 5 was modified to increase the likelihood of observing a strong congruency-byalerting interaction.

One aspect that seems to be crucial in this regard is the degree of dimensional overlap between the target and the flankers (see, e.g., Kornblum, Hasbroucq, \& Osman, 1990). Specifically, in the majority of studies reporting robust congruency-by-alerting interactions in the flanker task, the stimuli that served as flankers were of the same stimulus set as the target. For instance, Weinbach and Henik (2012, Exp. 1) used arrows as stimuli for both the target and flanker sets. In this experimental setup, the target and flankers do not overlap in a common spatial feature (as in Exp. 4 of the present study), but because they are perceptually identical and are directly related in terms of their associated responses. According to the dimensional-overlap model (Kornblum et al., 1990), this stronger overlap between the target and flankers should increase the likelihood of observing a solid congruency effect and, thereby, the potential to observe an interaction with alerting signals. Applying this logic to the present study, the identity of the flankers in Experiment 5 was changed so that both the target and flankers were letters, and therefore had stronger dimensional overlap.

Analogous to Experiment 4, I expected that the presentation of an alerting signal would increase the congruency effect in the main task, as reflected in a congruency-byalerting interaction. Furthermore, also analogous to Experiment 1, I expected that the alerting signal would exert a pronounced effect on probe RT, because it was presented directly in probe trials. Finally, and most importantly, I expected that the probe-RT function would be flattened by the presence of an alerting signal if alerting signals increased the size of the attentional focus. 


\section{Method}

Participants Thirty-eight participants (24 women, 14 men; $M_{\text {age }}=23.0$ years, age range: $19-37$ years) took part in Experiment 5. All of the participants had normal or corrected-to-normal visual acuity. They gave their written informed consent before the experiment and received either course credit or payment $(€ 12)$ for their participation.

Apparatus and stimuli The apparatus and stimuli were identical to those of Experiment 4, except for the following modifications: Instead of arrows, the uppercase letters "A" and "B" (height $\times$ width for both letters: $0.7 \times 0.7^{\circ}$ of visual angle; edge thickness: $0.1^{\circ}$ of visual angle) now served as flankers. Furthermore, the lowercase letters " $a$ " and " $b$ " (height $\times$ width: $0.5 / 0.7 \times 0.5^{\circ}$ of visual angle; edge thickness: $0.1^{\circ}$ of visual angle) now served as the targets. This modification was done for two reasons: First, the flankers were semantically identical to the targets (i.e., the letters "a" and "b"), and thus had stronger dimensional overlap, which should lead to a more pronounced congruency-by-alerting interaction. Second, the use of lowercase instead of uppercase letters for the targets assured at the same time that the targets and flankers would not be physically identical, which avoided the problem that identical targets and flankers might form a strong perceptual group in congruent trials. Importantly, such a grouping effect would prevent the establishment of a narrow focus of attention, which in turn is necessary to measure a pronounced $\mathrm{V}$ shape in the probe-RT function.

A final modification concerned the nature of the response key assignment: First, participants' hands were now positioned on a common response box with three keys, which was aligned to the center of the screen. Second, participants now responded with their left index and their right middle finger to targets in the main task, whereas they used the right index finger for responses to probes. This modification in the response key assignment was made in order to minimize the impact of a potential spatial congruency effect on the probeRT function (i.e., that participants - if using their left index finger to respond to probes - would be faster at leftward probe positions simply because of the spatial congruency between probe position and response effector).

Procedure The trial procedure was identical to that of Experiment 4, except for the following changes: First, analogously to Experiment 1, the main task and the probe task were now presented in separate trials. Second, in half of the probetask trials, an alerting signal preceded the probe by an SOA of $150 \mathrm{~ms}$.

Participants first completed three practice blocks with 12 , 10, and 17 trials, respectively. Again, the first block contained only the main task, the second block contained only the probe task, and the third block contained both tasks in combination.
Analogously to Experiments 3 and 4, participants then completed 680 experimental trials, with a short break after every 34 trials. Of the 680 experimental trials, 480 contained the main task, and 200 contained the probe task. The main-task trials were further subdivided according to the factors congruency and alerting signal, resulting in 120 trials per experimental condition. Likewise, the probe-task trials were subdivided according to the factors probe position and alerting signal, resulting in 20 trials per experimental condition.

\section{Results}

The practice trials were excluded prior to the data analysis. For the analysis of RT, trials with incorrect responses were excluded, as well as trials with RT below $150 \mathrm{~ms}$ or above $1,400 \mathrm{~ms}$ ( $M=0.2 \%$ in the main task and $M=0.06 \%$ in the probe task). Mean accuracy was $M=94.1 \%$ in the main task and $M=$ $95.7 \%$ in the probe task. All statistical analyses were conducted analogously to Experiment 4. The results for mean RT in the main and the probe task is depicted in Fig. 6.

The analysis of mean RT in the main task revealed shorter $\mathrm{RT}$ in trials with an alerting signal than in trials without an alerting signal, $F(1,37)=222.19, p<.001, \eta_{\mathrm{p}}{ }^{2}=.86$ (see Fig. 6 , upper panel). Furthermore, mean RT was shorter in congruent than in incongruent trials, $F(1,37)=68.14, p<.001, \eta_{\mathrm{p}}{ }^{2}=$ .65. As in Experiment 4, the size of this congruency effect $\left(\mathrm{RT}_{\text {incon }}\right.$ minus $\left.\mathrm{RT}_{\text {con }}\right)$ was larger in trials with an alerting signal $(M=34 \mathrm{~ms})$ than in trials without an alerting signal $(M=21 \mathrm{~ms}), F(1,37)=8.38, p=.006, \eta_{\mathrm{p}}{ }^{2}=.18$. The analysis of mean accuracy in the main task revealed no main effect of alerting signal, $F(1,37)=1.10, p=.30$. However, mean accuracy was affected by congruency, $F(1,37)=6.94, p=.01, \eta_{\mathrm{p}}^{2}$ $=.16$, and this main effect was further qualified by the interaction of alerting signal and congruency, $F(1,37)=4.35, p=$ $.04, \eta_{\mathrm{p}}{ }^{2}=.11$. A post-hoc analysis of this interaction revealed that mean accuracy in trials with an alerting signal was higher in the congruent condition $(M=95.1 \%)$ than in the incongruent condition $\left(M=92.5 \% ; p_{\text {Holm }}=.02\right)$. In contrast, mean accuracy in trials without an alerting signal did not differ between the congruent condition $(M=94.8 \%)$ and the incongruent condition $\left(M=94.1 \% ; p_{\text {Holm }}=.16\right)$.

The analysis of mean RT in the probe task revealed a main effect of probe position, $F(4,148)=18.59, p<.001, \eta_{\mathrm{p}}{ }^{2}=.33$, since RT clearly varied as a function of the probe's position (see Fig. 6, lower panel). Apart from the main effect of probe position, a main effect of alerting signal also emerged, $F(1$, $37)=484.65, p<.001, \eta_{\mathrm{p}}{ }^{2}=.93$, reflecting shorter RT in trials with an alerting signal than in trials without an alerting signal. In contrast to Experiment 1, the interaction of the two factors was not significant, $F(1,37)=1.72, p=.15$. The analysis of mean accuracy in the probe task revealed main effects of probe position, $F(4,148)=7.16, p<.001, \eta_{\mathrm{p}}{ }^{2}=.16$, and alerting signal, $F(1,37)=4.92, p=.03, \eta_{\mathrm{p}}{ }^{2}=.12$, as well as 


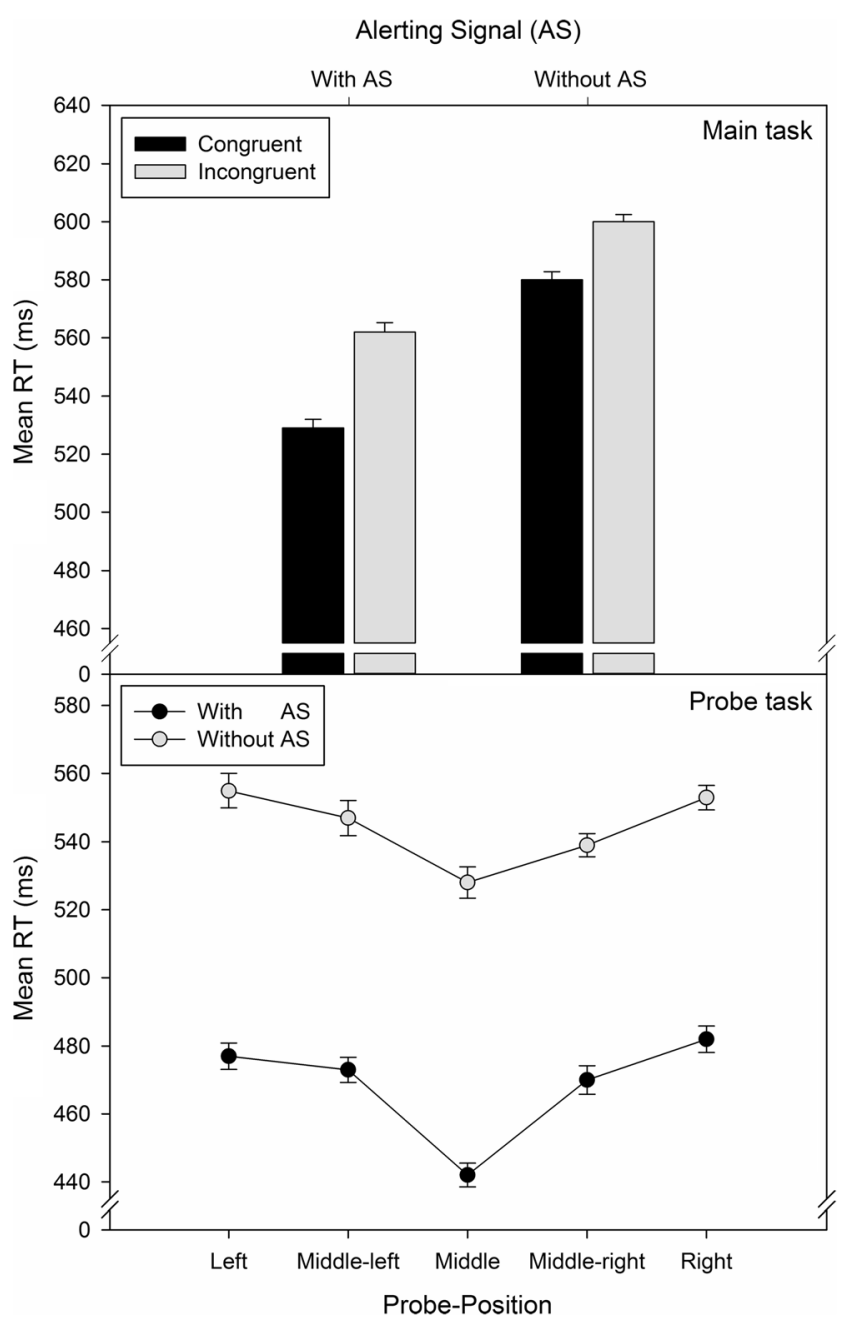

Fig. 6 Results of Experiment 5. (Upper panel) Mean reaction time (RT) in the main task as a function of alerting signal (AS) and congruency. (Lower panel) Mean RT in the probe task as a function of AS and probe position.

an interaction of the two factors, $F(4,148)=2.71, p=.03, \eta_{\mathrm{p}}{ }^{2}$ $=.07$. A post-hoc analysis of this interaction showed that mean accuracy in trials with an alerting signal was lower at the two leftward probe positions (i.e., $M=92.5 \%$ at the left position, and $M=92.6 \%$ at the middle-left position) than at the middle position $\left(M=98.0 \%\right.$; both $p_{\mathrm{Holm}} \mathrm{s}=<.004$; all other $p_{\text {Holm }} \mathrm{s}$. 17). This bias toward more errors at leftward probe positions was nearly absent in trials without an alerting signal, where accuracy differed only between the left position $(M=$ $94.9 \%)$ and the middle-right position $\left(M=97.8 \% ; p_{\text {Holm }}=\right.$ .04 ), but not between any of the other positions (all $p_{\mathrm{Holm}} \mathrm{s}>$ $.32)$.

Analogous to Experiments 1-4, the impact of the alerting signal on the shape of the probe-RT function was assessed by means of a trend analysis on standardized probe RT. This analysis revealed a quadratic trend for the effect of probe position, $F(1,37)=60.94, p<.001, \eta_{\mathrm{p}}{ }^{2}=.62$, reflecting an increase in RT toward lateral probe positions (see Fig. 6, lower panel). This quadratic trend, however, was not modulated by alerting signal, $F(1,37)=0.91, p=.35$. Apart from the quadratic trend, there was also a quartic trend for the effect of probe position, $F(1,37)=20.79, p<.001, \eta_{\mathrm{p}}{ }^{2}=.36$, and this trend was modulated by alerting signal, $F(1,37)=4.41, p=$ $.04, \eta_{\mathrm{p}}{ }^{2}=.11$. No further meaningful trends emerged for the effect of probe position (all $F_{\mathrm{S}}<3.4$ ), nor were there any modulations of these trends by alerting signal (all $F_{\mathrm{S}}<1.9$ ).

To investigate whether the interaction of alerting signals with the quartic trend was due to a specifically large RT effect at the middle probe position (see Exp. 1), I also compared the RT difference between each two neighboring probe positions between trials with and without an alerting signal. In contrast to Experiment 1, this analysis revealed only a marginally significant alerting effect for the RT difference between the middle and middle-right position $\left(p_{\mathrm{Holm}}=.06\right.$; all other $p_{\mathrm{Holm}} \mathrm{s}>$ .62).

Finally, I conducted an additional analysis in which I aimed to maximize the statistical power for detecting an alerting effect on the attentional focus by including data from both Experiments 1 and 5. Specifically, I performed a trend analysis on the standardized probe RT, with the within-subjects factors alerting signal (with alerting signal, without alerting signal) and probe position (left, middle-left, middle, middle-right, right) and the between-subjects factor experiment (Exp. 1, Exp. 5). I chose Experiments 1 and 5 because these experiments were the most comparable in terms of the trial procedure and because they had revealed a pronounced alerting effect on mean RT in the probe task. The combined analysis of the two experiments showed a quadratic trend for the effect of probe position, $F(1,72)=106.53, p<.001, \eta_{\mathrm{p}}{ }^{2}=.60$, as well as a quartic trend, $F(1,72)=24.51, p<.001, \eta_{\mathrm{p}}{ }^{2}=.25$. Again, however, the combined analysis did not reveal an interaction of alerting signal with the quadratic trend, $F(1,72)=2.15, p=$ .15 , only an interaction of alerting signal with the quartic trend, $F(1,72)=14.07, p<.001, \eta_{\mathrm{p}}{ }^{2}=.16$. There were no further meaningful trends (all $F \mathrm{~s}<3.2$ ). Furthermore, no two-way interaction of the factor experiment with any of the trends was observed (all $F \mathrm{~s}<1.85$ ), nor a three-way interaction of all factors (all $F \mathrm{~s}<1)$.

\section{Discussion}

As expected, Experiment 5 revealed solid congruency and alerting effects in the main task, since participants responded faster overall in congruent trials and in trials with an alerting signal. Second, and more importantly, Experiment 5 showed a pronounced congruency-by-alerting interaction: Specifically, the size of the congruency effect on RT was clearly larger in trials with than in trials without an alerting signal, which is in line with the results of Experiment 4 and previous studies (e.g., Fischer et al., 2010, 2012; Weinbach \& Henik, 2012). This increase in flanker interference was also mirrored in the 
pattern of results observed for mean accuracy in the main task: Specifically, participants responded less accurately in incongruent than in congruent trials, but only when an alerting signal had been presented beforehand. Third, Experiment 5 also revealed the expected probe-RT function (LaBerge, 1983): Specifically, RT in the probe task was fastest at the middle position and increased toward more lateral positions, as indexed by a main effect of probe position and a quadratic trend in the probe-RT function. ${ }^{4}$ This pattern of results shows that the attentional focus was again successfully captured in Experiment 5.

Even more importantly, Experiment 5 also revealed a pronounced alerting effect in the probe task: Specifically, participants responded faster overall to probes in trials with than in trials without an alerting signal. Yet, Experiment 5 again did not reveal a flattened probe-RT function in trials with an alerting signal. Specifically, the analysis of standardized probe RT showed that the quadratic trend-indexing the size of the attentional focus - did not vary as a function of alerting signal. Furthermore, no modulation of the quadratic trend was observed when I increased the statistical power by means of a combined analysis of the probe-RT functions in Experiments 1 and 5. Instead, the effect of the alerting signal on the probeRT function in Experiment 5 showed up in an interaction with the quartic trend in that function. Unlike in Experiment 1, however, this interaction was not due to a specifically large RT difference between the middle position and the middle-left (middle-right) position in trials with an alerting signal. Rather, this interaction reflected a more prominent folding of the probe-RT function at the middle-right probe position in trials with an alerting signal. Although the origin of this stronger folding is unclear, it does not change the interpretation of the major result - that is, the observation that alerting signals did not modulate the quadratic trend in the probe-RT function.

In sum, Experiment 5 shows that even an experimental setup that allowed to measure a solid congruency-by-alerting interaction and a direct alerting effect on probe RTs did not reveal a flattening of the quadratic trend in the probe-RT function. Hence, the results of Experiment 5 again do not provide

\footnotetext{
${ }^{4}$ It should be noted that accuracy in the probe task also varied as a function of the probe's position. Yet, this effect was specific to the leftward positions, whereas no meaningful accuracy differences emerged between the middle and rightward positions. One potential explanation for this reduction in accuracy at leftward positions could be that the specific response key assignment used in Experiment 5 (i.e., responding with the right index finger to probes) did not completely prevent the emergence of a spatial congruency effect. Specifically, because participants had to respond to probes with their right index finger, they may have made more errors when probes were presented to the left of fixation (and thus at a spatially incongruent location). Such an explanation, albeit speculative, would be consistent with the observation that the increase in the error rate for leftward positions was larger in trials with alerting signals, because alerting signals also increase spatial congruency effects (Böckler et al., 2011; Fischer et al., 2010). Importantly, this possibility does not alter the interpretation of the probe-RT function, because no accuracy differences were observed at rightward positions.
}

supporting evidence for the idea of an increased attentional focus underlying the congruency-by-alerting interaction.

\section{General discussion}

In a series of five experiments, I examined whether alerting signals increase the focus of visuospatial attention (Weinbach \& Henik, 2012). To this end, a paradigm was employed that has been shown to effectively measure variations in the size of the attentional focus (e.g., LaBerge, 1983; LaBerge \& Brown, 1986). In the main task, participants' focus of attention was anchored on a central target letter in a five-item display. In the probe task, the deployment of attention was then measured by means of a probe that was randomly presented at all five display positions. In this experimental setup, the attentional focus is indexed by differences in RT as a function of the probe's position (LaBerge, 1983; LaBerge \& Brown, 1986). Specifically, RT to probes presented at the central display position is shortest, since this is the position of the target in the majority of trials, and increases toward more lateral probe positions. As was outlined in the introduction, this RT pattern corresponds to a V-shaped probe-RT function and can be mathematically described in terms of a quadratic trend (LaBerge, 1983). Importantly, the slope of this quadratic trend should vary as a size of the attentional focus. Specifically, it should be less pronounced in the case of a wider attentional focus, because more attention resources would be allocated toward lateral positions. Hence, if alerting signals increase the size of the attentional focus, they should lead to a flatter probe-RT function, as reflected by a reduced quadratic trend.

At odds with this hypothesis, the results of the present experiments did not show any indication for a flattened probe-RT function in trials with an alerting signal. Specifically, although the probe-RT functions in all experiments clearly exhibited a quadratic trend, corresponding to a $\mathrm{V}$-shaped probe-RT function, this trend was not modulated by the presence of an alerting signal. It is unlikely that the absence of an alerting effect on the quadratic trend could be attributable to a lack of sensitivity: First, alerting signals affected probe RT, as reflected in faster mean RT in the probe task (Exps. 1,3, and 5). Second, alerting signals still had an impact on the shape of the probe-RT function, as reflected in the modulation of the quartic trend (Exps. 1,2, and 5). Third, even an experimental setup in which the alerting signal was presented immediately before the probe display (Exps. 1 and 5), and therefore captured the alerting effect without a preceding effect on the main task, did not show a flattened probe-RT function in alerting trials. It is furthermore unlikely that the modulation of the attentional focus depends on the presence of salient events in the spatial surround of the attended stimulus: Specifically, even when four identical stimuli were used as flankers (i.e., arrowheads in Exps. 3 and 4, and letters in Exp. 5), alerting signals did not flatten the quadratic RT trend. Finally, it is also 
unlikely that the absence of an influence on the attentional focus was due to a weak congruency-by-alerting interaction or a lack of statistical power: Specifically, Experiment 5 revealed a pronounced congruency-by-alerting interaction in the main task, as well as a strong alerting effect on RT in the probe task. Still, alerting signals did not flatten the probe-RT function in this experiment. In addition, even a combined analysis across Experiments 1 and 5, which served to increase the statistical power for observing an alerting effect on the probe-RT function, did not reveal the expected flattening of the probe-RT function. In sum, the consistent absence of an alerting effect on the quadratic trend in the probe-RT function clearly questions the idea that alerting signals increase congruency effects via an increased attentional focus. ${ }^{5}$

Apart from the present study, empirical evidence that is at odds with the idea of an increased attentional focus has been observed also in other studies. First of all, Weinbach and Henik (2014) reported results from a global-local task that are only partially consistent with the idea that alerting signals increase the attentional focus. As was explicated in the introduction, conflict in the global-local task is introduced between a global and a local feature of a hierarchical stimulus. Although the conflict induced by an irrelevant but incongruent global feature is typically stronger than the other way round, the effect of the local feature can be increased if it is made salient enough. Applying this logic, Weinbach and Henik (2014) manipulated the relative salience of global and local features and then measured the effect of alerting signals on the respective congruency effects. Interestingly, these authors observed that alerting signals increased conflict caused by both, the global and the local feature, depending on which feature was more salient. For instance, when participants responded to the global feature, alerting signals increased conflict caused by an incongruent, but more salient local feature. In contrast, when participants responded to the local feature, alerting signals increased conflict caused by an incongruent salient global feature. This pattern of results is not consistent with the idea that alerting signals per se increase the attentional focus. If alerting signals simply led to a larger focus of attention, they should always facilitate a global interpretation of a stimulus and thereby specifically increase the impact of the global feature on stimulus processing (see Weinbach \& Henik, 2011). Second, empirical evidence at odds with an increased attentional focus has also been reported by Wiegand et al. (2017). These authors used a computational modeling approach based on the theory of visual attention (TVA; Bundesen, 1990) to

\footnotetext{
${ }^{5}$ It should be noted that the idea of an increased attentional focus might still be preserved under specific assumptions (and I thank an anonymous reviewer for pointing this out). Specifically, even though Experiment 5 yielded a profound congruency-by-alerting interaction, it is nonetheless possible that alerting signals could modulate the attentional focus in experimental setups that would yield an even stronger congruency-by-alerting interaction. Such an additional restriction, however, undermines the idea of a widened attentional focus as a general mechanism that could explain the congruency-by-alerting interactions in different experimental tasks.
}

examine the influence of alerting signals on processing of a multi-element display. Interestingly, Wiegand et al. observed that alerting signals modulated TVA parameters reflecting the speed of perceptual processing, but did not affect TVA parameters indexing the distribution of spatial attention, as would have been expected if alerting signals increased the attentional focus. Presumably most challenging for the idea of a widened attentional focus, however, are the results of a study by Fischer et al. (2012). As was explicated in the introduction, these authors observed that the congruency effect in a word flanker task was only enhanced by alerting signals when the flanker words were drawn from the same set as the target words, but not when they were drawn from a categorically related set. This result can hardly be explained in terms of an increased attentional focus, because in this scenario a larger congruency effect should be observed irrespective of whether the flanker is drawn from the target set (and thus directly associated with a response) or not.

If the congruency-by-alerting interaction, as observed in the present study, is not driven by an increase in the attentional focus, the question arises of which other mechanism underlies this effect? As was explicated in the introduction, another prominent account in the literature locates the emergence of the congruency-by-alerting interaction at the level of response selection (e.g., Fischer et al., 2010, 2012). According to this account, alerting signals increase congruency effects because they facilitate the activation of automatic response tendencies that are based on a direct transmission of visual information into corresponding motor codes. To date, direct empirical evidence in support of this response activation account has been observed in an event-related potential (ERP) study showing that alerting signals directly modulate ERP indicators of response activation (Böckler et al., 2011) and in a behavioral study showing that the congruency-by-alerting interaction is observed when the flankers are drawn from the same stimulus set as the target and, thus, are directly associated with the target response (Fischer et al., 2012). Transferred to the present study, the response activation account can explain the congruency-byalerting interaction (as observed in Exps. 4 and 5) because the flankers were either (semantically) identical to the target set (Exp. 5), and therefore directly associated with the target response, or because the flankers contained a spatial feature that overlapped with the spatially defined target response (Exp. 4$)^{6}$

\footnotetext{
${ }^{6}$ An indirect response association of the arrow flankers with the target response in Experiment 4 can be assumed because automatic response activation is not necessarily bound to direct pairings of a stimulus with a response, but can also arise whenever an (irrelevant) feature of the stimulus is processed automatically and overlaps with the response set. Supporting evidence for this assumption has come from ERP studies showing that the mere presentation of an arrow leads to an activation of the corresponding response, as indexed by the LRP in the ERP (see, e.g., Eimer, 1995; Willemssen et al., 2004). Transferred to Experiment 4 of the present study, the spatial information conveyed by the arrow flankers would have automatically activated the corresponding spatial response (i.e., left or right) and therefore led to a congruency effect.
} 
Furthermore, the response activation account can explain why alerting signals did not modulate the quadratic trend in the probe-RT function, because it attributes the emergence of the congruency-by-alerting interaction to an influence on response selection, not attentional processes. Therefore, it does not predict variations in the quadratic trend.

Although the major results of the present study can be explained in terms of an influence of alerting signals on response selection - as is assumed within the response activation account —other aspects of the study suggest that alerting signals do not exclusively operate on response selection. First of these is the observation that alerting signals affected response speed in the main task of Experiment 2 and the probe tasks of Experiments 2-4 but did not lead to a general increase in error rates. Second, in both cases participants did not perform a classical response selection task, but a go-no-go task (the main task in Exp. 2) or a simple detection task (the probe task in Exps. 2-5). Here, it could be argued that the beneficial effect of alerting signals cannot be attributed solely to an influence on response selection, because there is no selection between two response options in these tasks (but see Gomez, Ratcliff, \& Perea, 2007, for evidence that go-no-go tasks may be a special variant of response selection tasks). Instead, an alerting effect on response speed without an increase in error rates might be better explained in terms of a perceptual processing benefit (see also Weinbach, Kalanthroff, Avnit, \& Henik, 2015). In line with this alternative explanation, a variety of studies investigating alerting effects outside of conflict paradigms have shown that alerting signals modulate indicators of perceptual processing. For instance, alerting signals have been shown to improve conscious perception of near-threshold stimuli (Kusnir, Chica, Mitsumasu, \& Bartolomeo, 2011) and to modulate early ERP components that are directly linked to perceptual processing (e.g., Böckler et al., 2011; Jepma, Wagenmakers, Band, \& Nieuwenhuis, 2009; Wiegand et al., 2017). Furthermore, in a functional imaging study, Fischer, Plessow, and Ruge (2013) have shown that alerting signals modulate neural activity in primary visual cortex, indicating that alerting signals may directly increase the efficiency of visual processing.

To accommodate for these perceptual effects, the response activation account could be expanded by the additional assumption that alerting signal initially affect perceptual processing, and this effect then transfers to response selection processes. Consistent with this idea, Fischer et al. (2013) more recently provided an extension of their response activation account: Specifically, on the basis of the above-mentioned observation that alerting signals modulate neural activity in primary visual cortex, these authors have suggested that alerting signals, in the first place, lead to more efficient processing of visual information. As a side effect, this beneficial effect on perceptual processing also leads to faster transmission of visual information into corresponding motor codes. In the case of conflict tasks, this will then increase the impact of the irrelevant stimuli (e.g., the flankers) on response selection (see also Fischer, Plessow, \& Kiesel, 2013). In this manner, the response activation account may also explain why alerting signals modulate response speed in paradigms that do not necessarily entail a response selection process, and why this benefit is not necessarily accompanied by an increase in error rates.

The idea that an alerting effect on perceptual processing plays a role in the congruency-by-alerting interaction in addition to its effect on response selection has been explicitly formulated in an account by Nieuwenhuis and de Kleijn (2013). These authors used a computational modeling approach to investigate why alerting signals increase congruency effects. Interestingly, they showed that the congruencyby-alerting interaction in a variety of conflict tasks (such as the Simon task and the flanker task) can be described by the interplay of two different factors: First, an alerting effect on the time required for stimulus encoding, an assumption that is rooted in the so-called early onset hypothesis (see, e.g., Rolke, 2008; Seibold, Bausenhart, Rolke, \& Ulrich, 2011); second, variations in the strength of cognitive control required for correct responding, which is initially weak in conflict tasks and requires some time to unfold its maximum effect. According to Nieuwenhuis and de Kleijn, a reduction of the time required to encode a stimulus will lead to an earlier onset of response selection, and thereby reduce the time to reach the decision threshold. If the threshold is reached early, however, cognitive control favoring selection of the correct response (i.e., the target response) is still weak. Therefore, response selection will be mainly determined by response information provided by the irrelevant stimulus (i.e., the flanker). Consequently, a larger congruency effect will be observed. Consistent with the assumption of a reduction in stimulus encoding time, several studies have shown that alerting signals directly modulate perceptual processing speed (Jepma et al., 2009; Matthias et al., 2010; Wiegand et al., 2017). Furthermore, consistent with the assumption that executive control requires some time to evolve in conflict tasks, several studies examining accuracy across the whole RT range have shown that fast responses tend to be specifically error prone in conflict tasks (e.g., Gratton, Coles, Sirevaag, Eriksen, \& Donchin, 1988; Ridderinkhof, 2002). Transferred to the present study, this early onset account can explain the observation of a larger congruency effect in trials with an alerting signal, because the assumed reduction of stimulus encoding time will cause an earlier onset of response selection. Furthermore, this account can explain the beneficial effect of alerting signals in the go-no-go task of Experiment 2, if one assumes that the reduction in stimulus encoding times would leave more time for the decision to 
respond (or not respond). ${ }^{7}$ Most importantly, it can also explain the lack of an alerting effect on the quadratic trend in the probe-RT function, because the congruency-by-alerting interaction is attributed to an influence on perceptual processing speed, not a change in the distribution of spatial attention. Hence, the early onset account provides a plausible alternative explanation of the results observed in the present study.

More recently, Weinbach and Henik (2014) have put forward another perceptual account of the congruency-byalerting interaction. As was mentioned above, these authors observed that alerting signals increased either global-to-local or local-to-global interference in a global-local task, depending on the salience of the global or local features. To explain this specific pattern of results, Weinbach and Henik (2014) have suggested that alerting signals bias attention toward salient stimuli (or stimulus features) in a visual display. This assumption is in line with models of visual attention that assume that attentional selection is not only determined by a topdown controlled mechanism that selects stimuli according to their task-relevance, but can also be determined by a fast bottom-up mechanism that selects stimuli according to their salience (e.g., Itti \& Koch, 2000). Accordingly, if alerting signals promote processing of salient stimulus features, these features will be selected for further processing and therefore will have a stronger impact on response selection in the global-local task. Although the global-local task is typically considered to measure the efficiency of level-based visual selection rather than spatial selection (see, e.g., Mevorach, Humphreys, \& Shalev, 2009), the logic that alerting signals promote the processing of salient events could also be transferred to the flanker task (see Weinbach \& Henik, 2014). Specifically, analogous to the more salient feature in the global-local task, the flankers can be considered to be more salient than the target, because they carry redundant information and may form a perceptual group. Hence, if alerting signals bias attention toward the more salient flankers, a larger congruency effect will emerge. Transferred to the present study, this salience account thus provides a plausible explanation for the congruency-by-alerting interaction observed in Experiments 4 and 5. Furthermore, this account can also explain why alerting signals facilitated performance in the go-no-go task of Experiment 2, because it explicitly assumes that alerting signals facilitate perceptual processing. Finally, the salience account may also explain why alerting signals did not modulate the quadratic trend in the probe-RT function: Specifically, with respect to Experiments 2-4, the salience account may explain this result if one assumes that the alerting effect had

\footnotetext{
${ }^{7}$ It should be noted that this explanation is only valid if one assumes that the initial level of cognitive control in the go-no-go task of Experiment 2 was already high, so that the reduced stimulus encoding time in trials with alerting signals did not result in more erroneous responses to no-go stimuli (see also Weinbach et al., 2015, for a discussion of this matter).
}

decayed already when probe was presented - due to the rather long SOA between the alerting signal and the probe. Therefore, an effect of increased attention toward the more salient flankers may not have shown up in the probe-RT function anymore. Furthermore, with respect to Experiments 1 and 5 , the salience account can explain this result, because an alerting signal was presented not only in the main task, but also in the probe task and, therefore, could directly affect processing of the probe. If one assumes that the salience of the probe does not vary across probe positions, an equal alerting effect should be observed across probe positions. In sum, the salience account provides another plausible perceptual account of the present results.

Yet, like a pure response activation account, a pure perceptual account such as the salience account is also open to criticism: First, such an account cannot directly explain why alerting signals - besides their effect on response speedcan also lead to an increase in error rates in go-no-go tasks, such as the probe task of Experiment 1 (but see Weinbach et al., 2015) and simple detection tasks (e.g., Posner et al., 1973; see also the probe task in Exp. 5). If alerting signals exclusively facilitated perceptual processing in these tasks (i.e., by biasing attention toward the most salient stimuli), they should not lead to an increase in error rates, because there is no conflicting stimulus information. Second, and in contrast to the response activation account, the salience account does not provide a stringent explanation for the observation that alerting signals increase congruency effects only when flankers are drawn from the same set as the target, not when they are merely categorically related to the target (Fischer et al., 2012). If alerting signals simply biased attention toward the flankers due their higher perceptual salience, it should not matter whether the flankers are drawn from the same set as the target or from a categorically related set. To accommodate this result, the salience account requires at least the additional assumption that not only stimulus-driven factors - such as the redundancy of the flanker features or their potential to form a perceptual group - but also top-down factors- such as a stronger weighting of flankers that are directly associated with the target response - contribute to the salience of the flankers. Hence, in this regard, the salience account requires a further specification of the factors that determine the higher salience of the flankers and how these factors interact with target processing in conflict tasks. Nonetheless, given that the salience account-like the response activation and early-onset accounts - can explain the congruency-by-alerting interaction and the absence of an alerting effect on the quadratic trend in the probe-RT function, it is also clear that the present study does not allow for deciding between the described accounts. Instead, further research will be required in order to test these accounts against each other.

To summarize, the main contribution of the present study is that it has provided a direct test of the hypothesis that alerting 
signals increase congruency effects in conflict tasks by increasing the size of the attentional focus. At variance with this hypothesis, the present study does not provide supporting evidence for an influence of alerting signals on the size of the attentional focus, even in the presence of a pronounced congruency-by-alerting interaction. Instead, the results of the present study are more consistent with nonspatial accounts that attribute the alerting effect to an influence on perceptual processing, which then leads to a larger congruency effect at the level of response selection.

Author note I thank Michael Hoyer, Lena Schön, Lydia Kastner, and Magdalena Król for their kind support in data acquisition. Furthermore, I thank Bettina Rolke for insightful discussions, and particularly for her valuable advice on a previous version of the manuscript. Finally, I thank an anonymous reviewer, Noam Weinbach, and Karla Evans for their helpful and constructive comments on a previous version of the manuscript.

\section{References}

Bernstein, I. H., Clark, M. H., \& Edelstein, B. A. (1969). Effects of an auditory signal on visual reaction time. Journal of Experimental Psychology, 80, 567-569. https://doi.org/10.1037/h0027444

Bertelson, P., \& Tisseyre, F. (1969). The time-course of preparation: Confirmatory results with visual and auditory warning signals. Acta Psychologica, 30, 145-154. https://doi.org/10.1016/00016918(69)90047-X

Böckler, A., Alpay, G., \& Stürmer, B. (2011). Accessory stimuli affect the emergence of conflict, not conflict control: A Simon-task ERP study. Experimental Psychology, 58, 102-109. https://doi.org/10. 1027/1618-3169/a000073

Bundesen, C. (1990). A theory of visual attention. Psychological Review, 97, 523-47. https://doi.org/10.1037/0033-295X.97.4.523

Callejas, A., Lupiáñez, J., Funes, M. J., \& Tudela, P. (2005). Modulations among the alerting, orienting, and executive control networks. Experimental Brain Research, 167, 23-37. https://doi.org/10.1007/ s00221-005-2365-Z

Callejas, A., Lupiáñez, J., \& Tudela, P. (2004). The three attentional networks: On their independence and interactions. Brain and Cognition, 54, 225-227. https://doi.org/10.1016/j.bandc.2004.02. 012

Carrasco, M. (2011). Visual attention: The past 25 years. Vision Research, 51, 1484-1525. https://doi.org/10.1016/j.visres.2011.04.012

de Jong, R., Liang, C.-C., \& Lauber, E. (1994). Conditional and unconditional automaticity: A dual-process model of effects of spatial stimulus-response correspondence. Journal of Experimental Psychology: Human Perception and Performance, 20, 731-750. https://doi.org/10.1037/0096-1523.20.4.731

R Development Core Team. (2016). R: A language and environment for statistical computing (Version 3.3.1) [Computer software]. Vienna: R Foundation for Statistical Computing. Retrieved from www.Rproject.org

Diamond, A. (2013). Executive functions. Annual Review of Psychology, 64, 135-168. https://doi.org/10.1146/annurev-psych-113011143750
Driver, J. (2001). A selective review of selective attention research from the past century. British Journal of Psychology, 92, 53-78. https:// doi.org/10.1348/000712601162103

Eimer, M. (1995). Stimulus-response compatibility and automatic response activation: Evidence from psychophysiological studies. Journal of Experimental Psychology: Human Perception and Performance, 21, 837-854. https://doi.org/10.1037/0096-1523.21. 4.837

Eriksen, B. A., \& Eriksen, C. W. (1974). Effects of noise letters upon the identification of a target letter in a nonsearch task. Perception \& Psychophysics, 16, 143-149. https://doi.org/10.3758/BF03203267

Fan, J., McCandliss, B. D., Sommer, T., Raz, A., \& Posner, M. I. (2002). Testing the efficiency and independence of attentional networks. Journal of Cognitive Neuroscience, 14, 340-347. https://doi.org/ 10.1162/089892902317361886

Fernandez-Duque, D., \& Posner, M. I. (1997). Relating the mechanisms of orienting and alerting. Neuropsychologia, 35, 477-486. https:// doi.org/10.1016/S0028-3932(96)00103-0

Fischer, R., Plessow, F., \& Kiesel, A. (2010). Auditory warning signals affect mechanisms of response selection: Evidence from a Simon task. Experimental Psychology, 57, 89-97. https://doi.org/10.1027/ 1618-3169/a000012

Fischer, R., Plessow, F., \& Kiesel, A. (2012). The effects of alerting signals in action control: Activation of S-R associations or inhibition of executive control processes? Psychological Research, 76, 317-328. https://doi.org/10.1007/s00426-011-0350-7

Fischer, R., Plessow, F., \& Kiesel, A. (2013). The effects of alerting signals in masked priming. Frontiers in Psychology, 4, 448. https://doi.org/10.3389/fpsyg.2013.00448

Fischer, R., Plessow, F., \& Ruge, H. (2013). Priming of visual cortex by temporal attention? The effects of temporal predictability on stimulus(-specific) processing in early visual cortical areas. NeuroImage, 66, 261-269. https://doi.org/10.1016/j.neuroimage.2012.10.091

Fischer, R., Schubert, T., \& Liepelt, R. (2007). Accessory stimuli modulate effects of nonconscious priming. Perception \& Psychophysics, 69, 9-22. https://doi.org/10.3758/BF03194449

Gomez, P. Ratcliff, R., \& Perea, M. (2007). A model of the go/no-go task. Journal of Experimental Psychology: General, 136, 389-413. https://doi.org/10.1037/0096-3445.136.3.389

Gratton, G., Coles, M. G. H., Sirevaag, E. J., Eriksen, C. W., \& Donchin, E. (1988). Pre- and poststimulus activation of response channels: A psychophysiological analysis. Journal of Experimental Psychology: Human Perception and Performance, 14, 331-344. https://doi.org/ 10.1037/0096-1523.14.3.331

Habekost, T., Petersen, A., \& Vangkilde, S. (2014). Testing attention: Comparing the ANT with TVA-based assessment. Behavior Research Methods, 46, 81-94. https://doi.org/10.3758/s13428-0130341-2

Hackley, S. A., \& Valle-Inclán, F. (1998). Automatic alerting does not speed late motoric processes in a reaction-time task. Nature, 391, 786-788. https://doi.org/10.1038/35849

Holm, S. (1979). A simple sequentially rejective multiple test procedure. Scandinavian Journal of Statistics, 6, 65-70.

Hommel, B. (1993). Spontaneous decay of response-code activation. Psychological Research, 56, 261-268. https://doi.org/10.1007/ BF00419656

Itti, L., \& Koch, C. (2000). A saliency-based search mechanism for overt and covert shifts of visual attention. Vision Research, 40, 14891506. https://doi.org/10.1016/S0042-6989(99)00163-7

James, W. (1890). Principles of psychology (vol. 1). New York: Holt.

Jepma, M., Wagenmakers, E.-J., Band, G. P., \& Nieuwenhuis, S. (2009). The effects of accessory stimuli on information processing: Evidence from electrophysiology and a diffusion model analysis. Journal of Cognitive Neuroscience, 21, 847-864. https://doi.org/ 10.1162/jocn.2009.21063 
Keuss, P. J. G. (1972). Reaction time to the second of two shortly spaced auditory signals both varying in intensity. Acta Psychologica, 36, 226-238. https://doi.org/10.1016/0001-6918(72)90007-8

Kornblum, S., Hasbroucq, T., \& Osman, A. (1990). Dimensional overlap: Cognitive basis for stimulus-response compatibility-A model and taxonomy. Psychological Review, 97, 253-270. https://doi.org/10. 1037/0033-295X.97.2.253

Kusnir, F., Chica, A. B., Mitsumasu, M. A., \& Bartolomeo, P. (2011). Phasic auditory alerting improves visual conscious perception. Consciousness and Cognition, 20, 1201-1210. https://doi.org/10. 1016/j.concog.2011.01.012

LaBerge, D. (1983). Spatial extent of attention to letters and words. Journal of Experimental Psychology: Human Perception and Performance, 9, 371-379. https://doi.org/10.1037/0096-1523.9.3. 371

LaBerge, D., \& Brown, V. (1986). Variations in size of the visual field in which targets are presented: An attentional range effect. Perception \& Psychophysics, 40, 188-200. https://doi.org/10.3758/ BF03203016

Matthias, E., Bublak, P., Müller, H. J., Schneider, W. X., Krummenacher, J., \& Finke, K. (2010). The influence of alertness on spatial and nonspatial components of visual attention. Journal of Experimental Psychology: Human Perception and Performance, 36, 38-56. https://doi.org/10.1037/a0017602

Mevorach, C., Humphreys, G. W., \& Shalev, L. (2009). Reflexive and preparatory selection and suppression of salient information in the right and left posterior parietal cortex. Journal of Cognitive Neuroscience, 21, 1204-1214. https://doi.org/10.1162/jocn.2009. 21088

Miller, J. (1991). The flanker compatibility effect as a function of visual angle, attentional focus, visual transients, and perceptual load: A search for boundary conditions. Perception \& Psychophysics, 49, 270-288. https://doi.org/10.3758/BF03214311

Morey, R. D. (2008). Confidence intervals from normalized data: A correction to Cousineau (2005). Tutorials in Quantitative Methods for Psychology, 4, 61-64.

Nieuwenhuis, S., \& de Kleijn, R. (2013). The impact of alertness on cognitive control. Journal of Experimental Psychology: Human Perception and Performance, 39, 1797-1801. https://doi.org/10. 1037/a0033980

Petersen, S. E., \& Posner, M. I. (2012). The attention system of the human brain: 20 years after. Annual Review of Neuroscience, 35, 73-89. https://doi.org/10.1146/annurev-neuro-062111-150525

Posner, M. I. (1980). Orienting of attention. Quarterly Journal of Experimental Psychology, 32, 3-25.

Posner, M. I. (1994). Attention: The mechanisms of consciousness. Proceedings of the National Academy of Sciences, USA, 91, 73987403. https://doi.org/10.1073/pnas.91.16.7398

Posner, M. I., \& Boies, S. J. (1971). Components of attention. Psychological Review, 78, 391-408. https://doi.org/10.1037/ h0031333

Posner, M. I., Klein, R., Summers, J., \& Buggie, S. (1973). On the selection of signals. Memory \& Cognition, 1, 2-12. https://doi.org/10. 3758/BF03198062

Posner, M. I., \& Petersen, S. E. (1990). The attention system of the human brain. Annual Review of Neuroscience, 13, 25-42. https://doi.org/ 10.1146/annurev.ne.13.030190.000325

Posner, M. I., Snyder, C. R., \& Davidson, B. J. (1980). Attention and the detection of signals. Journal of Experimental Psychology, 109, 160 174. https://doi.org/10.1037/0096-3445.109.2.160
Raz, A., \& Buhle, J. (2006). Typologies of attentional networks. Nature Reviews Neuroscience, 7, 367-379. https://doi.org/10.1038/nrn1903

Ridderinkhof, K. R. (2002). Activation and suppression in conflict tasks: Empirical clarification through distributional analyses. In W. Prinz \& B. Hommel (Eds.), Attention and performance XIX (pp. 494 519). Oxford: Oxford University Press.

Ristic, J., Friesen, C. K., \& Kingstone, A. (2002). Are eyes special? It depends on how you look at it. Psychonomic Bulletin \& Review, 9, 507-513. https://doi.org/10.3758/BF03196306

Rolke, B. (2008). Temporal preparation facilitates perceptual identification of letters. Perception \& Psychophysics, 70, 1305-1313. https:// doi.org/10.3758/PP.70.7.1305

Seibold, V. C., Bausenhart, K. M., Rolke, B., \& Ulrich, R. (2011). Does temporal preparation increase the rate of sensory information accumulation? Acta Psychologica, 137, 56-64. https://doi.org/10.1016/j. actpsy.2011.02.006

Simon, J. R., \& Rudell, A. P. (1967). Auditory S-R compatibility: The effect of an irrelevant cue on information processing. Journal of Applied Psychology, 81, 174-176. https://doi.org/10.1037/ h0020586

Stroop, S. R. (1935). Studies of interference in serial verbal reactions. Journal of Experimental Psychology, 18, 643-662. https://doi.org/ 10.1037/h0054651

Tipples, J. (2002). Eye gaze is not unique: Automatic orienting in response to uninformative arrows. Psychonomic Bulletin \& Review, 9, 314-318. https://doi.org/10.3758/BF03196287

Ulrich, R., \& Mattes, S. (1996). Does immediate arousal enhance response force in simple reaction time? Quarterly Journal of Experimental Psychology, 49A, 972-990. https://doi.org/10.1080/ 713755672

Weinbach, N., \& Henik, A. (2011). Phasic alertness can modulate executive control by enhancing global processing of visual stimuli. Cognition, 121, 454-458. https://doi.org/10.1016/j.cognition.2011. 08.010

Weinbach, N., \& Henik, A. (2012). The relationship between alertness and executive control. Journal of Experimental Psychology: Human Perception and Performance, 38, 1530-1540. https://doi.org/10. 1037/a0027875

Weinbach, N., \& Henik, A. (2013). The interaction between alerting and executive control: Dissociating phasic arousal and temporal expectancy. Attention, Perception, \& Psychophysics, 75, 1374-1381. https://doi.org/10.3758/s13414-013-0501-6

Weinbach, N., \& Henik, A. (2014). Alerting enhances attentional bias for salient stimuli: Evidence from a global/local processing task. Cognition, 133, 414-419. https://doi.org/10.1016/j.cognition.2014. 07.005

Weinbach, N., Kalanthroff, E., Avnit, A., \& Henik, A. (2015). Can arousal modulate response inhibition? Journal of Experimental Psychology: Learning, Memory, and Cognition, 41, 1873-1877. https://doi.org/10.1037/xlm0000118

Wiegand, I., Petersen, A., Finke, K., Bundesen, C., Lansner, J., \& Habekost, T. (2017). Behavioral and brain measures of phasic alerting effects on visual attention. Frontiers in Human Neuroscience, 11, 176. https://doi.org/10.3389/fnhum.2017.00176

Willemssen, R., Hoormann, J., Hohnsbein, J., \& Falkenstein, M. (2004). Central and parietal event-related lateralizations in a flanker task. Psychophysiology, 41, 762-771. https://doi.org/10.1111/j.14698986.2004.00201.x 